\title{
Dynamics of annihilation. II. Fluctuations of global quantities
}

\author{
Pablo Maynar, ${ }^{1,2}$ María Isabel García de Soria, ${ }^{3}$ Grégory Schehr, ${ }^{1}$ Alain Barrat, ${ }^{1}$ and Emmanuel Trizac ${ }^{3}$ \\ ${ }^{1}$ Laboratoire de Physique Théorique (CNRS UMR 8627), Bâtiment 210, Université Paris-Sud, 91405 Orsay Cedex, France \\ ${ }^{2}$ Física Teórica, Universidad de Sevilla, Apartado de Correos 1065, E-41080, Sevilla, Spain \\ ${ }^{3}$ LPTMS (CNRS UMR 8626), Université Paris-Sud, Orsay Cedex, F-91405, France
}

(Received 17 January 2008; published 28 May 2008)

\begin{abstract}
We develop a theory for fluctuations and correlations in a gas evolving under ballistic annihilation dynamics. Starting from the hierarchy of equations governing the evolution of microscopic densities in phase space, we subsequently restrict our attention to a regime of spatial homogeneity, and obtain explicit predictions for the fluctuations and time correlation of the total number of particles, total linear momentum, and total kinetic energy. Cross correlations between these quantities are worked out as well. These predictions are successfully tested against molecular dynamics and Monte Carlo simulations. This provides strong support for the theoretical approach developed, including the hydrodynamic treatment of the spectrum of the linearized Boltzmann operator. This paper makes use of the spectral analysis reported in the preceding paper [Phys. Rev. E 77, 051127 (2008)]
\end{abstract}

DOI: 10.1103/PhysRevE.77.051128

PACS number(s): 02.50.-r, 51.10.+y, 05.20.Dd, 82.20.Nk

\section{INTRODUCTION}

Systems where particles may react, change chemical or physical nature, and ultimately disappear, model a rich variety of phenomena and provide prominent situations to develop and test the foundations of nonequilibrium statistical mechanics (see, e.g., [2-6] and references therein). When reactions are controlled ballistically, the system can be modeled by an assembly of hard spheres or disks which annihilate with probability $p$ or collide elastically with probability $1-p$ every time two particles meet each other [7]. Within the framework of this probabilistic ballistic annihilation (PBA) model, most of the work carried out up to now has focused on the kinetic equations for the one-body distribution function and the information following from them [7-11]. In particular, the hydrodynamic equations, with explicit expressions for the transport coefficients, have been derived by using a generalization of the Chapman-Enskog expansion [12]. The preceding paper [1], where we have established the hydrodynamic description in the context of the eigenfunctions and eigenvalues of the linearized Boltzmann collision operator, falls in this vein. Within this formalism, the conditions in which the hydrodynamic description is expected to apply are somewhat more transparent, and can be expressed in terms of some properties of the linearized Boltzmann collision operator.

In the present paper, the goal is to go beyond the study of one-body quantities; the focus is on fluctuations and correlations. To this end, we use the tools and ideas developed in the context of the linearized Boltzmann equation. The dynamical behavior of the correlations in the dilute limit can indeed be obtained in terms of the linearized Boltzmann collision operator. The study of correlations in the PBA model allows one to go beyond the description at the level of average values, and to characterize how global magnitudes (such as the total number of particles or the total energy) fluctuate around their average. It has already been shown in other classes of dissipative systems, such as in granular systems, that the knowledge of fluctuations is relevant in order to understand the behavior of the system when vortices or clusters develop [13,14], or even in simpler situations where the system is still homogeneous $[15,16]$. The main goal of this paper is to formulate a theory of fluctuations for the PBA model in the dilute limit and to apply it to one of the simplest possible states, namely, the homogeneous decay state, exploiting its scaling properties. This will allow us to obtain explicit expressions for the distributions characterizing the velocity correlations in the system, and to compute the statistics of the total number of particles, total momentum, and total energy, which decrease monotonically due to the annihilation process.

The paper is organized as follows. In Sec. II, we present the general framework of the hierarchy method [17] which allows us to write the evolution equations of correlation functions. The specific case of the homogeneous decay state is considered in Sec. III, where the scaling properties of this state are used to simplify the equations. After briefly recalling in Sec. IV how fluctuations and correlations of global observables can be computed from the knowledge of the two-particle correlation functions, we first consider in Sec. V the correlation functions at equal time, which give access to the fluctuations of the total number of particles, total momentum, and total energy. We obtain theoretical predictions for the asymptotic scaling regime as well as for the shorttime behavior, and we test these predictions against numerical simulations (both molecular dynamics and Monte Carlo). In Sec. VI, we generalize our results to the two-time correlation functions and compare also to numerical simulations. Finally, Sec. VII contains some discussions of the results and our conclusions. For the sake of readability, this paper contains some overlap with its companion [1]. Repetitions have been kept to a minimum though, and we therefore refer to [1] for several technical details.

\section{GENERAL FRAMEWORK}

The system we consider consists of a dilute gas of identical smooth hard spheres or disks of mass $m$ and diameter $\sigma$, 
moving ballistically in dimension $d$. The particles are supposed to undergo only binary collisions. When two particles collide they annihilate with probability $p$ or collide elastically with probability $1-p$. In this probabilistic ballistic annihilation, there is therefore no conserved quantity (except for $p=0$ ) and the number of particles decreases steadily. In this section, we will show how to obtain evolution equations for the correlation functions in this system. The general idea of the method is to derive a closed set of equations for the distribution functions describing the fluctuations by using the same kind of approximations as needed to derive the kinetic equation, in our case the Boltzmann equation. In this way, a unified formalism provides the usual kinetic equation as well as evolution equations for the one- and two-time correlations.

Let $X_{j} \equiv\left\{\mathbf{R}_{j}(t), \mathbf{V}_{j}(t)\right\}$ denote the position and velocity of particle $j$ in the system at time $t$. Both, $\mathbf{R}_{j}(t)$ and $\mathbf{V}_{j}(t)$ are parametric functions of the initial positions and velocities of all particles. Microscopic one- and two-particle densities in the phase space are defined by

$$
\begin{gathered}
F_{1}\left(x_{1}, t\right)=\sum_{i=1}^{N} \delta\left[x_{1}-X_{i}(t)\right], \\
F_{2}\left(x_{1}, x_{2}, t\right)=\sum_{i=1}^{N} \sum_{i \neq j}^{N} \delta\left[x_{1}-X_{i}(t)\right] \delta\left[x_{2}-X_{j}(t)\right],
\end{gathered}
$$

and higher-order functions can similarly be defined. Here and in the following, the lower-case variables $x_{i} \equiv\left\{\mathbf{r}_{i}, \mathbf{v}_{i}\right\}$ are field variables referring to a particular point in phase space.

The initial state of the system is characterized by a point in phase space, $\Gamma \equiv\left\{X_{1}, \ldots, X_{N}\right\}$, which is chosen at random according to a probability $\rho(\Gamma, 0)$. When the notation $\langle G\rangle$ $\equiv \int d \Gamma G(\Gamma) \rho(\Gamma, 0)$ is introduced for the average over the initial conditions, the averages of the microscopic densities $F_{s}\left(x_{1}, \ldots, x_{s}, t\right)$ over $\rho(\Gamma, 0)$ are the usual one-time reduced distribution functions

$$
f_{s}\left(x_{1}, \ldots, x_{s}, t\right)=\left\langle F_{s}\left(x_{1}, \ldots, x_{s}, t\right)\right\rangle .
$$

Similarly, two-time reduced distribution functions can also be defined in terms of the microscopic densities as

$$
\begin{aligned}
f_{r, s} & \left(x_{1}, \ldots, x_{r}, t ; x_{1}^{\prime}, \ldots, x_{s}^{\prime}, t^{\prime}\right) \\
& =\left\langle F_{r}\left(x_{1}, \ldots, x_{r}, t\right) F_{s}\left(x_{1}^{\prime}, \ldots, x_{s}^{\prime}, t^{\prime}\right)\right\rangle .
\end{aligned}
$$

For simplicity we will consider $t>t^{\prime}>0$ in the following.

We now introduce the two-particle correlation functions through the usual cluster expansion. The one-time correlation function $g_{2}$ and the two-time correlation function $h_{1,1}$ are then defined by

$$
\begin{aligned}
f_{2}\left(x_{1}, x_{2}, t\right) & =f_{1}\left(x_{1}, t\right) f_{1}\left(x_{2}, t\right)+g_{2}\left(x_{1}, x_{2}, t\right), \\
f_{1,1}\left(x_{1}, t ; x_{2}, t^{\prime}\right) & =f_{1}\left(x_{1}, t\right) f_{1}\left(x_{2}, t^{\prime}\right)+h_{1,1}\left(x_{1}, t ; x_{2}, t^{\prime}\right) .
\end{aligned}
$$

It is easy to show from the definitions of $f_{1}, f_{2}$, and $f_{1,1}$ that

$$
h_{1,1}\left(x_{1}, t ; x_{2}, t\right)=g_{2}\left(x_{1}, x_{2}, t\right)+\delta\left(x_{1}-x_{2}\right) f_{1}\left(x_{1}, t\right) .
$$

The case of deterministic annihilation $(p=1)$ was considered in Ref. [10]. The hierarchy of equations for the reduced dis- tribution functions is then shown to be similar to the one describing elastic collisions, once the binary elastic collision operator is replaced by the operator describing annihilating collisions. In the PBA case, assuming molecular chaos, i.e., that no correlations exist between colliding particles, the equation for $f_{1}\left(x_{1}, t\right)$ is the Boltzmann equation

$$
\left(\frac{\partial}{\partial t}+L^{(0)}\left(x_{1}\right)\right) f_{1}\left(x_{1}, t\right)=J\left[f_{1}, f_{1}\right],
$$

where

$$
\begin{gathered}
L^{(0)}\left(x_{1}\right)=\mathbf{v}_{1} \cdot \frac{\partial}{\partial \mathbf{r}_{1}}, \\
J\left[f_{1}, f_{1}\right]=\int d x_{2} \delta\left(\mathbf{r}_{12}\right) \bar{T}_{0}\left(\mathbf{v}_{1}, \mathbf{v}_{2}\right) f_{1}\left(x_{1}, t\right) f_{1}\left(x_{2}, t\right),
\end{gathered}
$$

and

$$
\begin{aligned}
\bar{T}_{0}\left(\mathbf{v}_{1}, \mathbf{v}_{2}\right)= & \sigma^{d-1} \int d \hat{\boldsymbol{\sigma}} \Theta\left(\mathbf{v}_{12} \cdot \hat{\boldsymbol{\sigma}}\right)\left(\mathbf{v}_{12} \cdot \hat{\boldsymbol{\sigma}}\right) \\
& \times\left[(1-p)\left(b_{\sigma}^{-1}-1\right)-p\right],
\end{aligned}
$$

is the PBA binary collision operator. In the above expressions $\mathbf{v}_{12}=\mathbf{v}_{1}-\mathbf{v}_{2}$ is the relative velocity, $\mathbf{r}_{12}=\mathbf{r}_{1}-\mathbf{r}_{2}$ the relative position, $\Theta$ the Heaviside function, $\hat{\boldsymbol{\sigma}}$ a unit vector joining the centers of two particles at collision, and $b_{\sigma}^{-1}$ an operator that replaces all the velocities $\mathbf{v}_{1}$ and $\mathbf{v}_{2}$ appearing to its right by the precollisional values $\mathbf{v}_{1}^{*}$ and $\mathbf{v}_{2}^{*}$,

$$
\begin{aligned}
& b_{\sigma}^{-1} \mathbf{v}_{1} \equiv \mathbf{v}_{1}^{*}=\mathbf{v}_{1}-\left(\mathbf{v}_{12} \cdot \hat{\boldsymbol{\sigma}}\right) \hat{\boldsymbol{\sigma}}, \\
& b_{\sigma}^{-1} \mathbf{v}_{2} \equiv \mathbf{v}_{2}^{*}=\mathbf{v}_{2}+\left(\mathbf{v}_{12} \cdot \hat{\boldsymbol{\sigma}}\right) \hat{\boldsymbol{\sigma}} .
\end{aligned}
$$

The equation for the correlation function $g_{2}$ can be obtained under the same hypothesis required to derive the Boltzmann equation, following the same lines as in Ref. [18] in the case of inelastically colliding particles, as

$$
\begin{aligned}
& \left(\frac{\partial}{\partial t}+L^{(0)}\left(x_{1}\right)+L^{(0)}\left(x_{2}\right)-K\left(x_{1}, t\right)-K\left(x_{2}, t\right)\right) g_{2}\left(x_{1}, x_{2}, t\right) \\
& =\delta\left(\mathbf{r}_{12}\right) \bar{T}_{0}\left(\mathbf{v}_{1}, \mathbf{v}_{2}\right) f_{1}\left(x_{1}, t\right) f_{1}\left(x_{2}, t\right),
\end{aligned}
$$

where we have introduced the linear operator $K\left(x_{i}, t\right)$,

$$
K\left(x_{i}, t\right)=\int d x_{3} \delta\left(\mathbf{r}_{i 3}\right) \bar{T}_{0}\left(\mathbf{v}_{i}, \mathbf{v}_{3}\right)\left(1+\mathcal{P}_{i 3}\right) f_{1}\left(x_{3}, t\right),
$$

and where the permutation operator $\mathcal{P}_{a b}$ interchanges the labels of particles $a$ and $b$ in the quantities on which it acts.

Finally, the evolution equation for $h_{1,1}$ reads

$$
\left(\frac{\partial}{\partial t}+L^{(0)}\left(x_{1}\right)-K\left(x_{1}, t\right)\right) h_{1,1}\left(x_{1}, t ; x_{2}, t^{\prime}\right)=0, \quad t>t^{\prime},
$$

which has to be solved with the initial condition (7), $h_{1,1}\left(x_{1}, t^{\prime} ; x_{2}, t^{\prime}\right)=g_{2}\left(x_{1}, x_{2}, t^{\prime}\right)+\delta\left(x_{1}-x_{2}\right) f_{1}\left(x_{1}, t^{\prime}\right)$.

The equations for the correlation functions $h_{1,1}$ and $g_{2}$ contain a part corresponding to free streaming and another 
one that corresponds to collisions. In particular, the evolution of the one-time correlation function $g_{2}$ due to collisions has two parts: one due to collisions of particle 1 or 2 (corresponding to the indices of the correlation function) with a third particle, which is governed by the Boltzmann collision operator; and a second one, due to collisions of particle 1 with particle 2, which can be written in terms of the oneparticle distribution function as a consequence of the molecular chaos hypothesis. In fact, as in the case of the inelastic granular gas [18], the only difference between the evolution equations of the correlation functions for the PBA and for a system of elastic particles lies in the substitution of the elastic binary collision operator by the operator for the PBA model, $\bar{T}_{0}\left(\mathbf{v}_{1}, \mathbf{v}_{2}\right)$. However, this does not give any $a$ priori guarantee on the range of validity of these equations. This prescription, i.e., how small the density of the system must be so that the above kinetic equations provide an accurate description, might depend on the parameter $p$, and also on the particular state being considered.

We will see in Sec. IV how the knowledge of $h_{1,1}$ and $g_{2}$ allows us to obtain the correlation functions of any observable that is a function of the particle positions in phase space.

\section{HOMOGENEOUS DECAY STATE}

As recalled in the preceding paper [1], the Boltzmann equation for the PBA model (8) admits a particular solution $f_{H}(\mathbf{v}, t)$ describing a spatially homogeneous decay state (HDS), in which all the time dependence is contained in the evolution of the density $n_{H}(t)$ and the temperature $T_{H}(t)$, which are defined as in standard kinetic theory as

$$
n_{H}(t)=\int d \mathbf{v} f_{H}(\mathbf{v}, t), \quad \frac{d}{2} n_{H}(t) T_{H}(t)=\int d \mathbf{v} \frac{m}{2} v^{2} f_{H}(\mathbf{v}, t) .
$$

Although there exists no rigorous proof of its stability nor of the fact that such a state should be approached from arbitrary initial conditions, numerical results obtained by molecular dynamic simulations and by the direct Monte Carlo method strongly support the existence of such a homogeneous solution [7,9]. In this section, we review for completeness the evolution equation of the one-particle distribution function and obtain the equations for adequately rescaled correlation functions. All quantities concerning this homogeneous decay state will be labeled by an index $H$.

In the HDS, the one-body distribution function does not depend on space and follows the scaling form [10]

$$
f_{H}(\mathbf{v}, t)=\frac{n_{H}(t)}{v_{H}^{d}(t)} \chi_{H}(\mathbf{c}),
$$

where $n_{H}(t)$ is the uniform density, $v_{H}(t)$ is the thermal (rootmean-square) velocity related to the granular temperature $T_{H}(t)$ by

$$
v_{H}(t)=\left(\frac{2 T_{H}(t)}{m}\right)^{1 / 2},
$$

and $\chi(\mathbf{c})$ is an isotropic function depending only on the modulus $c=|\mathbf{c}|$ of the rescaled velocity $\mathbf{c}=\mathbf{v} / v_{H}(t)$. Moreover, the density and temperature fields evolve according to [12]

$$
\begin{aligned}
& \frac{d n_{H}(t)}{d t}=-p \nu_{H}(t) \zeta_{n} n_{H}(t), \\
& \frac{d T_{H}(t)}{d t}=-p \nu_{H}(t) \zeta_{T} T_{H}(t),
\end{aligned}
$$

where $\nu_{H}(t)$ is the collision frequency of the corresponding hard-sphere fluid in equilibrium (with same temperature and density)

$$
\nu_{H}(t)=\frac{n_{H}(t) T_{H}^{1 / 2}(t) \sigma^{d-1}}{m^{1 / 2}} \frac{8 \pi^{d-1 / 2}}{(d+2) \Gamma(d / 2)},
$$

and the dimensionless decay rates $\zeta_{n}$ and $\zeta_{T}$ are functionals of the distribution function:

$$
p \zeta_{n}=-\frac{\gamma}{2} \int d \mathbf{c}_{1} \int d \mathbf{c}_{2} T\left(\mathbf{c}_{1}, \mathbf{c}_{2}\right) \chi_{H}\left(\mathbf{c}_{1}\right) \chi_{H}\left(\mathbf{c}_{2}\right)
$$

$$
p \zeta_{T}=-\frac{\gamma}{2} \int d \mathbf{c}_{1} \int d \mathbf{c}_{2}\left(\frac{2 c_{1}^{2}}{d}-1\right) T\left(\mathbf{c}_{1}, \mathbf{c}_{2}\right) \chi_{H}\left(\mathbf{c}_{1}\right) \chi_{H}\left(\mathbf{c}_{2}\right) .
$$

In these expressions, $\gamma$, which does not depend on time, is given by $\gamma=2 n_{H}(t) v_{H}(t) \sigma^{d-1} / \nu_{H}(t)=(d+2) \sqrt{2} \Gamma(d / 2) /$ $\left(4 \pi^{(d-1) / 2}\right)$, and the binary collision operator $T\left(\mathbf{c}_{1}, \mathbf{c}_{2}\right)$ takes the form

$$
T\left(\mathbf{c}_{1}, \mathbf{c}_{2}\right)=\int d \hat{\boldsymbol{\sigma}} \Theta\left(\mathbf{c}_{12} \cdot \hat{\boldsymbol{\sigma}}\right)\left(\mathbf{c}_{12} \cdot \hat{\boldsymbol{\sigma}}\right)\left[(1-p) b_{\sigma}^{-1}-1\right] .
$$

Finally, the scaled distribution function $\chi_{H}(\mathbf{c})$ obeys the equation

$$
\begin{array}{r}
p\left[\left(d \zeta_{T}-2 \zeta_{n}\right)+\zeta_{T} \mathbf{c}_{1} \cdot \frac{\partial}{\partial \mathbf{c}_{1}}\right] \chi_{H}\left(\mathbf{c}_{1}\right) \\
\quad=\gamma \int d \mathbf{c}_{2} T\left(\mathbf{c}_{1}, \mathbf{c}_{2}\right) \chi_{H}\left(\mathbf{c}_{1}\right) \chi_{H}\left(\mathbf{c}_{2}\right) .
\end{array}
$$

The operator $b_{\sigma}^{-1}$ in the last equation is defined again by Eqs. (12) and (13), but substituting $\left(\mathbf{v}_{1}, \mathbf{v}_{2}\right)$ by $\left(\mathbf{c}_{1}, \mathbf{c}_{2}\right)$. The analytical form of $\chi_{H}$ is not known, but its behavior at large and small velocities has been determined $[9,10]$. As in the preceding paper [1], we will use here the approximate form of the distribution function in the so-called first Sonine approximation, which is valid for velocities in the thermal region, and all the functionals of $\chi_{H}(\mathbf{c})$, like the decay rates and the transport coefficients, will be evaluated in this approximation $[7,9]$.

Considering the scaling form for the one-particle distribution function, it is convenient to introduce the rescaled correlation function $\widetilde{g}_{H}$ through 


$$
g_{2, H}\left(x_{1}, x_{2}, t\right)=\frac{n_{H}(t)}{v_{H}^{2 d}(t)} \widetilde{g}_{H}\left(\tau, \mathbf{r}_{12}, \mathbf{c}_{1}, \mathbf{c}_{2}\right)
$$

where we have taken into account that the system is invariant under space translation, so that $g_{2, H}$ depends on $\mathbf{r}_{12}=\mathbf{r}_{1}-\mathbf{r}_{2}$ and not on $\mathbf{r}_{1}$ and $\mathbf{r}_{2}$ separately. The dimensionless time scale $\tau$,

$$
\tau=\frac{1}{2} \int_{0}^{t} d t^{\prime} \nu_{H}\left(t^{\prime}\right)
$$

is proportional to the number of collisions in the time interval $[0, t]$. The equation for the reduced function $\widetilde{g}_{H}$ in these units reads then

$$
\begin{aligned}
& \left(-\frac{\partial}{\partial \tau}+\Lambda\left(\mathbf{c}_{1}\right)+\Lambda\left(\mathbf{c}_{2}\right)-2 p \zeta_{n}-l_{H}(\tau) \mathbf{c}_{12} \cdot \frac{\partial}{\partial \mathbf{r}_{12}}\right) \\
& \quad \times \widetilde{g}_{H}\left(\tau, \mathbf{r}_{12}, \mathbf{c}_{1}, \mathbf{c}_{2}\right)=-\delta\left(\mathbf{r}_{12}\right) \gamma T\left(\mathbf{c}_{1}, \mathbf{c}_{2}\right) \chi_{H}\left(\mathbf{c}_{1}\right) \chi_{H}\left(\mathbf{c}_{2}\right),
\end{aligned}
$$

where we have also introduced the length scale $l_{H}(t)$ $=2 v_{H}(t) / \nu_{H}(t)$, which is proportional to the instantaneous mean free path, and the linearized Boltzmann operator (see previous paper)

$$
\begin{aligned}
\Lambda\left(\mathbf{c}_{i}\right) h\left(\mathbf{c}_{i}\right)= & \gamma \int d \mathbf{c}_{3} T\left(\mathbf{c}_{i}, \mathbf{c}_{3}\right)\left(1+\mathcal{P}_{i 3}\right) \chi_{H}\left(\mathbf{c}_{3}\right) h\left(\mathbf{c}_{i}\right) \\
& +p\left(2 \zeta_{n}-d \zeta_{T}\right) h\left(\mathbf{c}_{i}\right)-p \zeta_{T} \mathbf{c}_{i} \cdot \frac{\partial}{\partial \mathbf{c}_{i}} h\left(\mathbf{c}_{i}\right)
\end{aligned}
$$

Similarly, we define a rescaled two-time correlation function $\tilde{h}_{H}$ through

$$
h_{1,1, H}\left(x_{1}, t ; x_{2}, t^{\prime}\right)=\frac{n_{H}(t)}{v_{H}^{d}(t) v_{H}^{d}\left(t^{\prime}\right)} \tilde{h}_{H}\left(\mathbf{r}_{12} ; \mathbf{c}_{1}, \tau ; \mathbf{c}_{2}, \tau^{\prime}\right),
$$

and obtain the following evolution equation:

$$
\begin{aligned}
& \frac{\partial}{\partial \tau} \tilde{h}_{H}\left(\mathbf{r}_{12} ; \mathbf{c}_{1}, \tau ; \mathbf{c}_{2}, \tau^{\prime}\right) \\
& \quad=\left(\Lambda\left(\mathbf{c}_{1}\right)-l_{H}(\tau) \mathbf{c}_{1} \cdot \frac{\partial}{\partial \mathbf{r}_{1}}\right) \tilde{h}_{H}\left(\mathbf{r}_{12} ; \mathbf{c}_{1}, \tau ; \mathbf{c}_{2}, \tau^{\prime}\right),
\end{aligned}
$$

with

$$
\tilde{h}_{H}\left(\mathbf{r}_{12} ; \mathbf{c}_{1}, \tau ; \mathbf{c}_{2}, \tau\right)=\tilde{g}_{H}\left(\tau, \mathbf{r}_{12}, \mathbf{c}_{1}, \mathbf{c}_{2}\right)+\delta\left(\mathbf{c}_{1}-\mathbf{c}_{2}\right) \delta\left(\mathbf{r}_{12}\right) \chi_{H}\left(\mathbf{c}_{1}\right) .
$$

It is interesting to note how, in this representation, all the time dependence due to the reference state is absorbed in the free streaming term through the function $l_{H}(\tau)$, proportional to the mean free path. The evolution of the correlation functions, moreover, will be determined by the properties of the linearized Boltzmann operator $\Lambda$, which we have already studied in the preceding paper [1] and which we will recall in Sec. V.

\section{FROM PARTICLE CORRELATION FUNCTIONS TO CORRELATIONS AND FLUCTUATIONS OF GLOBAL MAGNITUDES}

In this section, we will show how the previously presented framework for correlation functions will allow us to study the fluctuations and correlations of global quantities for a PBA system in the homogeneous decay state. In particular, we will focus on the total number of particles $N$, the total momentum $\mathbf{P}$, and the total energy $E$.

Consider indeed two dynamical variables of the form

$$
\begin{aligned}
& A[\Gamma(t)]=\sum_{i=1}^{N} a\left(\mathbf{V}_{i}\right)=\int d x_{1} a\left(\mathbf{v}_{1}\right) F_{1}\left(x_{1}, t\right), \\
& B[\Gamma(t)]=\sum_{i=1}^{N} b\left(\mathbf{V}_{i}\right)=\int d x_{2} b\left(\mathbf{v}_{2}\right) F_{1}\left(x_{2}, t\right),
\end{aligned}
$$

where $a$ and $b$ are functions of the particle velocities $\mathbf{V}_{i}$, and $F_{1}\left(x_{1}, t\right)$ is the microscopic density in phase space (1). Taking $a=1, a=\mathbf{v}$, and $a=m \mathbf{v}^{2} / 2$ yields for $A$ the total number of particles $N$, the total momentum $\mathbf{P}$, and the total kinetic energy $E$, respectively. The deviations $\delta A(t)=A(t)-\langle A(t)\rangle_{H}$ and $\delta B(t)=B(t)-\langle B(t)\rangle_{H}$ of $A$ or $B$ from their average values in the HDS (denoted by $\langle\cdots\rangle_{H}$ ), define the fluctuations of both magnitudes, which have average zero and correlations

$$
\left\langle\delta A(t) \delta B\left(t^{\prime}\right)\right\rangle_{H}=\left\langle A(t) B\left(t^{\prime}\right)\right\rangle_{H}-\langle A(t)\rangle_{H}\left\langle B\left(t^{\prime}\right)\right\rangle_{H} .
$$

It is then straightforward to use the definition of the two-time correlation function $h_{1,1}$ in Eq. (6), to obtain

$$
\begin{aligned}
\left\langle\delta A(t) \delta B\left(t^{\prime}\right)\right\rangle_{H}= & \int d \mathbf{r}_{1} \int d \mathbf{v}_{1} \int d \mathbf{r}_{2} \int d \mathbf{v}_{2} a\left(\mathbf{v}_{1}\right) b\left(\mathbf{v}_{2}\right) \\
& \times h_{1,1, H}\left(\mathbf{r}_{1}, \mathbf{v}_{1}, t ; \mathbf{r}_{2}, \mathbf{v}_{2}, t^{\prime}\right) .
\end{aligned}
$$

In particular, for $t=t^{\prime}$, this leads to

$$
\begin{aligned}
\langle\delta A(t) \delta B(t)\rangle_{H}= & V \int d \mathbf{v} a(\mathbf{v}) b(\mathbf{v}) f_{H}(\mathbf{v}, t)+V \int d \mathbf{v}_{1} \int d \mathbf{v}_{2} \\
& \times a\left(\mathbf{v}_{1}\right) b\left(\mathbf{v}_{2}\right) \int d \mathbf{r}_{12} g_{2, H}\left(\mathbf{r}_{12}, \mathbf{v}_{1}, \mathbf{v}_{2}, t\right),
\end{aligned}
$$

where $V=\int d \mathbf{r}_{1}$ is the total volume of the system. These formulas show how the correlations of two different global magnitudes are determined by the one-particle distribution function and by the correlation functions. The one-particle distribution function is known in the HDS in the first Sonine approximation $[7,9]$.

\section{FLUCTUATIONS IN THE HDS}

Let us focus in this section on the one-time correlation function $\tilde{g}_{H}$. We will only need functions $a$ and $b$ which depend on the velocity degrees of freedom, so that it is convenient to integrate out the spatial dependence by introducing

$$
\phi_{H}\left(\tau, \mathbf{c}_{1}, \mathbf{c}_{2}\right) \equiv \int d \mathbf{r}_{12} \widetilde{g}_{H}\left(\tau, \mathbf{r}_{12}, \mathbf{c}_{1}, \mathbf{c}_{2}\right)
$$

whose evolution is obtained from (29) as 


$$
\begin{gathered}
\left(-\frac{\partial}{\partial \tau}+\Lambda\left(\mathbf{c}_{1}\right)+\Lambda\left(\mathbf{c}_{2}\right)-2 p \zeta_{n}\right) \phi_{H}\left(\tau, \mathbf{c}_{1}, \mathbf{c}_{2}\right) \\
=-\gamma T\left(\mathbf{c}_{1}, \mathbf{c}_{2}\right) \chi_{H}\left(\mathbf{c}_{1}\right) \chi_{H}\left(\mathbf{c}_{2}\right) .
\end{gathered}
$$

Given an initial condition $\phi_{H}\left(0, \mathbf{c}_{1}, \mathbf{c}_{2}\right)$, this Eq. (38) can be formally integrated as

$$
\begin{aligned}
\phi_{H}\left(\tau, \mathbf{c}_{1}, \mathbf{c}_{2}\right) & \\
= & e^{\left[\Lambda\left(\mathbf{c}_{1}\right)+\Lambda\left(\mathbf{c}_{2}\right)-2 p \zeta_{n}\right] \tau} \phi_{H}\left(0, \mathbf{c}_{1}, \mathbf{c}_{2}\right)+\int_{0}^{\tau} d \tau^{\prime} \\
& \times e^{\left(\tau-\tau^{\prime}\right)\left[\Lambda\left(\mathbf{c}_{1}\right)+\Lambda\left(\mathbf{c}_{2}\right)-2 p \zeta_{n}\right]} \gamma T\left(\mathbf{c}_{1}, \mathbf{c}_{2}\right) \chi_{H}\left(\mathbf{c}_{1}\right) \chi_{H}\left(\mathbf{c}_{2}\right) \\
= & e^{\left[\Lambda\left(\mathbf{c}_{1}\right)+\Lambda\left(\mathbf{c}_{2}\right)-2 p \zeta_{n}\right] \tau}\left[\phi_{H}\left(0, \mathbf{c}_{1}, \mathbf{c}_{2}\right)-\phi_{H}^{s}\left(\mathbf{c}_{1}, \mathbf{c}_{2}\right)\right]+\phi_{H}^{s}\left(\mathbf{c}_{1}, \mathbf{c}_{2}\right),
\end{aligned}
$$

where $\phi_{H}^{s}\left(\mathbf{c}_{1}, \mathbf{c}_{2}\right)$ is the solution of

$$
\left[\Lambda\left(\mathbf{c}_{1}\right)+\Lambda\left(\mathbf{c}_{2}\right)-2 p \zeta_{n}\right] \phi_{H}^{s}\left(\mathbf{c}_{1}, \mathbf{c}_{2}\right)=-\gamma T\left(\mathbf{c}_{1}, \mathbf{c}_{2}\right) \chi_{H}\left(\mathbf{c}_{1}\right) \chi_{H}\left(\mathbf{c}_{2}\right),
$$

where we implicitly assumed that $\Lambda$ is invertible. This happens to be the case; see below. The spectral properties of the linearized Boltzmann operator $\Lambda$ are thus crucial for the evaluation of $\phi_{H}$. We therefore start by recalling these properties.

\section{A. Spectral properties of $\Lambda$}

In our companion paper [1], we have analyzed the eigenvalue problem associated with the linearized Boltzmann operator $\Lambda$,

$$
\Lambda(\mathbf{c}) \xi_{\beta}(\mathbf{c})=\lambda_{\beta} \xi_{\beta}(\mathbf{c}) .
$$

We have in fact restricted ourselves to the hydrodynamic part of $\Lambda$, defined by those eigenvalues of the balance equations for the number density, momentum, and temperature following from the homogeneous linearized Boltzmann equation. Such eigenvalues are [12]

$$
\lambda_{1}=0, \quad \lambda_{2}=-p\left(\zeta_{T}+2 \zeta_{n}\right), \quad \lambda_{3}=p \zeta_{T} .
$$

Although we were not able to prove that these eigenvalues are indeed the hydrodynamic ones, the self-consistency of the resulting description and the successful comparison with numerical simulations have validated this assumption [1]. In the previous paper we also obtained the corresponding eigenfunctions

$$
\begin{gathered}
\xi_{1}(\mathbf{c})=\chi_{H}\left(\mathbf{c}_{1}\right)+\frac{\partial}{\partial \mathbf{c}} \cdot\left[\mathbf{c} \chi_{H}(\mathbf{c})\right], \\
\xi_{2}(\mathbf{c})=z \chi_{H}(\mathbf{c})-\frac{\partial}{\partial \mathbf{c}} \cdot\left[\mathbf{c} \chi_{H}(\mathbf{c})\right], \\
\boldsymbol{\xi}_{3}(\mathbf{c})=-\frac{\partial \chi_{H}(\mathbf{c})}{\partial \mathbf{c}},
\end{gathered}
$$

with $z=\frac{2 \zeta_{n}}{\zeta_{T}}$ a function of the probability of annihilation, $p$. The eigenvalue $\lambda_{3}$ is $d$-fold degenerate and we denote by $\xi_{3 i}$, $i=1, \ldots, d$, the corresponding eigenvectors. The scalar product of two functions $f(\mathbf{c})$ and $g(\mathbf{c})$ is defined as

$$
\langle f \mid g\rangle \equiv \int d \mathbf{c} \chi_{H}^{-1}(\mathbf{c}) f^{*}(\mathbf{c}) g(\mathbf{c}),
$$

$f^{*}$ being the complex conjugate of $f$. The eigenfunctions $\xi_{\beta}$ given in (43)-(45) are not orthogonal, as a consequence of the operator $\Lambda$ being non-Hermitian in the associated Hilbert space. On the other hand, it is easily verified that the set of functions $\left\{\bar{\xi}_{1} ; \bar{\xi}_{2} ; \bar{\xi}_{3}\right\}=\left\{\chi_{H}(\mathbf{c})-\frac{z}{1+z}\left(\frac{1}{2}+\frac{c^{2}}{d}\right) \chi_{H}(\mathbf{c})\right.$; $\left.\frac{1}{1+z}\left(\frac{1}{2}+\frac{c^{2}}{d}\right) \chi_{H}(\mathbf{c}) ; \mathbf{c} \chi_{H}(\mathbf{c})\right\}$ satisfy the biorthogonality condition $\left\langle\bar{\xi}_{\beta} \mid \xi_{\beta^{\prime}}\right\rangle=\delta_{\beta, \beta^{\prime}}$, for $\beta, \beta^{\prime}=1,2,3$.

The eigenfunctions of the operator $\left[\Lambda\left(\mathbf{c}_{1}\right)+\Lambda\left(\mathbf{c}_{2}\right)-2 p \zeta_{n}\right]$ that appear in Eq. (40) are then easily seen to be $\xi_{\beta_{1}}\left(\mathbf{c}_{1}\right) \xi_{\beta_{2}}\left(\mathbf{c}_{2}\right)$, with

$$
\begin{aligned}
& {\left[\Lambda\left(\mathbf{c}_{1}\right)+\Lambda\left(\mathbf{c}_{2}\right)-2 p \zeta_{n}\right] \xi_{\beta_{1}}\left(\mathbf{c}_{1}\right) \xi_{\beta_{2}}\left(\mathbf{c}_{2}\right)} \\
& \quad=\left(\lambda_{\beta_{1}}+\lambda_{\beta_{2}}-2 p \zeta_{n}\right) \xi_{\beta_{1}}\left(\mathbf{c}_{1}\right) \xi_{\beta_{2}}\left(\mathbf{c}_{2}\right)
\end{aligned}
$$

Since $\zeta_{n}>\zeta_{T}[12]$, and under the assumption that the norms of the "nonhydrodynamic" eigenvalues are always greater than the hydrodynamic ones, the eigenvalues of $\left[\Lambda\left(\mathbf{c}_{1}\right)\right.$ $\left.+\Lambda\left(\mathbf{c}_{2}\right)-2 p \zeta_{n}\right]$ are therefore all negative. This has the important consequence that the exponential term in (39) decays to zero and that the large-time limit of $\phi_{H}\left(\tau, \mathbf{c}_{1}, \mathbf{c}_{2}\right)$ is $\phi_{H}^{s}\left(\mathbf{c}_{1}, \mathbf{c}_{2}\right)$, the solution of Eq. (40).

\section{B. Hydrodynamic part of the correlation functions}

Obtaining the full spectrum of $\Lambda$ is a formidable task. We will here assume, as in the preceding paper [1], that the kinetic (nonhydrodynamic) modes have a fast decay, and work in the hydrodynamic subspace spanned by the functions $\xi_{\beta}$ defined in the previous section. With that purpose, we generalize the definition of the scalar product given in (46) to two-velocity functions by

$$
\begin{aligned}
& \left\langle f\left(\mathbf{c}_{1}, \mathbf{c}_{2}\right) \mid g\left(\mathbf{c}_{1}, \mathbf{c}_{2}\right)\right\rangle \\
& \quad \equiv \int d \mathbf{c}_{1} \int d \mathbf{c}_{2} \chi_{H}^{-1}\left(\mathbf{c}_{1}\right) \chi_{H}^{-1}\left(\mathbf{c}_{2}\right) f^{*}\left(\mathbf{c}_{1}, \mathbf{c}_{2}\right) g\left(\mathbf{c}_{1}, \mathbf{c}_{2}\right) .
\end{aligned}
$$

This allows us to define a projector operator $P_{12}$ onto the space spanned by the functions $\xi_{\beta_{1}}\left(\mathbf{c}_{1}\right) \xi_{\beta_{2}}\left(\mathbf{c}_{2}\right)$ as

$$
\begin{aligned}
& P_{12} f\left(\mathbf{c}_{1}, \mathbf{c}_{2}\right) \\
& \quad \equiv \sum_{\beta_{1}=1}^{3} \sum_{\beta_{2}=1}^{3}\left\langle\bar{\xi}_{\beta_{1}}\left(\mathbf{c}_{1}\right) \bar{\xi}_{\beta_{2}}\left(\mathbf{c}_{2}\right) \mid f\left(\mathbf{c}_{1}, \mathbf{c}_{2}\right)\right\rangle \xi_{\beta_{1}}\left(\mathbf{c}_{1}\right) \xi_{\beta_{2}}\left(\mathbf{c}_{2}\right),
\end{aligned}
$$

and the hydrodynamic parts of $\phi_{H}\left(\tau, \mathbf{c}_{1}, \mathbf{c}_{2}\right)$ and $\phi_{H}^{s}\left(\mathbf{c}_{1}, \mathbf{c}_{2}\right)$ are by definition

$$
\begin{aligned}
\phi_{H}^{(h)}\left(\tau, \mathbf{c}_{1}, \mathbf{c}_{2}\right) & \equiv P_{12} \phi_{H}\left(\tau, \mathbf{c}_{1}, \mathbf{c}_{2}\right) \\
& =\sum_{\beta_{1}, \beta_{2}=1}^{3} a_{\beta_{1}, \beta_{2}}(\tau) \xi_{\beta_{1}}\left(\mathbf{c}_{1}\right) \xi_{\beta_{2}}\left(\mathbf{c}_{2}\right),
\end{aligned}
$$




$$
\phi_{H}^{s(h)}\left(\mathbf{c}_{1}, \mathbf{c}_{2}\right) \equiv P_{12} \phi_{H}^{s}\left(\mathbf{c}_{1}, \mathbf{c}_{2}\right)=\sum_{\beta_{1}, \beta_{2}=1}^{3} a_{\beta_{1}, \beta_{2}}^{s} \xi_{\beta_{1}}\left(\mathbf{c}_{1}\right) \xi_{\beta_{2}}\left(\mathbf{c}_{2}\right) .
$$

We can now obtain a closed equation for $\phi_{H}^{(h)}$ by applying the operator $P_{12}$ on both sides of Eqs. (39) and (40), under the additional assumption that

$$
P_{12} \Lambda\left(\mathbf{c}_{i}\right)=P_{12} \Lambda\left(\mathbf{c}_{i}\right) P_{12} .
$$

A theoretical estimation a priori of the accuracy of this approximation would require more knowledge than is available at present about the spectrum of $\Lambda(\mathbf{c})$ and its adjoint. Therefore, it will be taken as a working hypothesis, to be validated later on by comparing the predictions it leads to with the results from numerical simulations of the system. It is worth emphasizing that, since $\Lambda$ leaves the hydrodynamic subspace invariant, Eq. (52) is equivalent to the commutation relation $P_{12} \Lambda=\Lambda P_{12}$. Proceeding further, we obtain from (39)

$$
\begin{aligned}
\phi_{H}^{(h)}( & \left.\tau, \mathbf{c}_{1}, \mathbf{c}_{2}\right) \\
= & e^{\left[\Lambda\left(c_{1}\right)+\Lambda\left(c_{2}\right)-2 p \zeta_{n}\right] \tau} P_{12}\left[\phi_{H}\left(0, \mathbf{c}_{1}, \mathbf{c}_{2}\right)-\phi_{H}^{s}\left(\mathbf{c}_{1}, \mathbf{c}_{2}\right)\right] \\
& +P_{12} \phi_{H}^{s}\left(\mathbf{c}_{1}, \mathbf{c}_{2}\right) \\
= & \sum_{\beta_{1}, \beta_{2}=1}^{3}\left(A_{\beta_{1}, \beta_{2}} e^{\left(\lambda_{\beta_{1}}+\lambda_{\beta_{2}}-2 p \zeta_{n}\right) \tau}+a_{\beta_{1}, \beta_{2}}^{s}\right) \xi_{\beta_{1}}\left(\mathbf{c}_{1}\right) \xi_{\beta_{2}}\left(\mathbf{c}_{2}\right),
\end{aligned}
$$

where we have introduced $A_{\beta_{1}, \beta_{2}}=a_{\beta_{1}, \beta_{2}}(0)-a_{\beta_{1}, \beta_{2}}^{s}$. We show in Appendix A how to obtain explicit formulas for the coefficients $a_{\beta_{1}, \beta_{2}}^{s}$ in terms of the cooling rates $\zeta_{n}$ and $\zeta_{T}$ and other coefficients, which are also functionals of the one-time distribution function $\chi_{H}$. The values of $a_{\beta_{1}, \beta_{2}}(0)$ depend on the initial condition $\phi_{H}\left(0, \mathbf{c}_{1}, \mathbf{c}_{2}\right)$. For the specific case in which the variables $N, \mathbf{P}$, and $E$ do not fluctuate at $t=0$, and taking into account that the system is in the HDS, the coefficients $a_{\beta_{1}, \beta_{2}}(0)$ are calculated in Appendix B.

\section{Hydrodynamic approximation for global fluctuations}

In this section we compute the correlation functions of the global observables by replacing $\phi_{H}$ implicitly appearing in (36) by its hydrodynamic part $\phi_{H}^{(h)}$. This can be done by invoking the relation

$\left\langle\bar{\xi}_{\beta_{1}}\left(\mathbf{c}_{1}\right) \bar{\xi}_{\beta_{2}}\left(\mathbf{c}_{2}\right) \mid f\left(\mathbf{c}_{1}, \mathbf{c}_{2}\right)\right\rangle=\left\langle\bar{\xi}_{\beta_{1}}\left(\mathbf{c}_{1}\right) \bar{\xi}_{\beta_{2}}\left(\mathbf{c}_{2}\right) \mid f^{(h)}\left(\mathbf{c}_{1}, \mathbf{c}_{2}\right)\right\rangle$,

for $\beta_{i}=1,2,3$. However, it must be stressed that the theoretical prediction for $\phi_{H}^{(h)}$ in Eq. (53) has been calculated using the approximation (52).

If we substitute $a(\mathbf{v})=1$ and $b(\mathbf{v})=1$ in (36), we obtain for the fluctuations of the number of particles

$$
\left\langle\delta N^{2}(\tau)\right\rangle_{H}=N_{H}(\tau)\left[\int d \mathbf{c} \chi_{H}(\mathbf{c})+\int d \mathbf{c}_{1} \int d \mathbf{c}_{2} \phi_{H}^{(h)}\left(\tau, \mathbf{c}_{1}, \mathbf{c}_{2}\right)\right],
$$

where we have introduced the notation $N_{H} \equiv\langle N\rangle_{H}$. In order to calculate the fluctuations of the total momentum we substitute $a(\mathbf{v})=v_{i}$ and $b(\mathbf{v})=v_{j}$ in Eq. (36) and obtain

$$
\begin{aligned}
\left\langle\delta P_{i}(\tau) \delta P_{j}(\tau)\right\rangle_{H}= & N_{H}(\tau) v_{H}^{2}(\tau)\left[\int d \mathbf{c} c_{i} c_{j} \chi_{H}(\mathbf{c})\right. \\
& \left.+\int d \mathbf{c}_{1} \int d \mathbf{c}_{2} c_{1 i} c_{2 j} \phi_{H}^{(h)}\left(\tau, \mathbf{c}_{1}, \mathbf{c}_{2}\right)\right] .
\end{aligned}
$$

For the energy we substitute $a(\mathbf{v})=b(\mathbf{v})=\frac{1}{2} m v^{2}$ so that we have

$$
\begin{aligned}
\left\langle\delta E^{2}(\tau)\right\rangle_{H}= & \frac{m^{2}}{4} N_{H}(\tau) v_{H}^{4}(\tau)\left[\int d \mathbf{c} c^{4} \chi_{H}(\mathbf{c})\right. \\
& \left.+\int d \mathbf{c}_{1} \int d \mathbf{c}_{2} c_{1}^{2} c_{2}^{2} \phi_{H}^{(h)}\left(\tau, \mathbf{c}_{1}, \mathbf{c}_{2}\right)\right] .
\end{aligned}
$$

Finally, we can calculate the correlation between $\delta N$ and $\delta E$ by taking $a(\mathbf{v})=1$ and $b(\mathbf{v})=\frac{1}{2} m v^{2}$

$$
\begin{aligned}
\langle\delta N(\tau) \delta E(\tau)\rangle_{H}= & \frac{m}{2} N_{H}(\tau) v_{H}^{2}(\tau)\left[\int d \mathbf{c} c^{2} \chi_{H}(\mathbf{c})\right. \\
& \left.+\int d \mathbf{c}_{1} \int d \mathbf{c}_{2} c_{2}^{2} \phi_{H}^{(h)}\left(\tau, \mathbf{c}_{1}, \mathbf{c}_{2}\right)\right] .
\end{aligned}
$$

After some algebra, we obtain

$$
\begin{aligned}
\left\langle\delta N^{2}(\tau)\right\rangle_{H}= & N_{H}(\tau)\left(1+a_{1,1}^{s}+2 z a_{1,2}^{s}+z^{2} a_{2,2}^{s}+A_{1,1} e^{-2 p \zeta_{n} \tau}\right. \\
& \left.+2 z A_{1,2} e^{-p\left(\zeta_{T}+4 \zeta_{n}\right) \tau}+z^{2} A_{2,2} e^{-2 p\left(\zeta_{T}+3 \zeta_{n}\right) \tau}\right), \quad(59) \\
\left\langle\delta P_{i}(\tau) \delta P_{j}(\tau)\right\rangle_{H}= & \delta_{i j} N_{H}(\tau) v_{H}^{2}(\tau)\left(\frac{1}{2}+a_{3 i, 3 i}^{s}\right)\left(1-e^{-2 p\left(\zeta_{n}-\zeta_{T}\right) \tau}\right), \\
\left\langle\delta E^{2}(\tau)\right\rangle_{H}= & \frac{m^{2}}{4} N_{H}(\tau) v_{H}^{4}(\tau)\left[\frac{d(d+2)}{4}\left(1+a_{2}\right)+\frac{d^{2}}{4} a_{1,1}^{s}\right. \\
& -d^{2}\left(1+\frac{z}{2}\right) a_{1,2}^{s}+d^{2}\left(1+\frac{z}{2}\right)^{2}+\frac{d^{2}}{4} A_{1,1} e^{-2 p \zeta_{n} \tau} \\
& -d^{2}\left(1+\frac{z}{2}\right) A_{1,2} e^{-p\left(\zeta_{T}+4 \zeta_{n}\right) \tau} \\
& \left.+d^{2}\left(1+\frac{z}{2}\right)^{2} A_{2,2} e^{-2 p\left(\zeta_{T}+3 \zeta_{n}\right) \tau}\right],
\end{aligned}
$$

and

$$
\begin{aligned}
\langle\delta N(\tau) \delta E(\tau)\rangle_{H}= & \frac{m}{2} N_{H}(\tau) v_{H}^{2}(\tau)\left[\frac{d}{2}-\frac{d}{2} a_{1,1}^{s}+d a_{1,2}^{s}\right. \\
& +d z\left(1+\frac{z}{2}\right) a_{2,2}^{s}-\frac{d}{2} A_{1,1} e^{-2 p \zeta_{n} \tau} \\
& +d A_{1,2} e^{-p\left(\zeta_{T}+4 \zeta_{n}\right) \tau} \\
& \left.+d z\left(1+\frac{z}{2}\right) A_{2,2} e^{-2 p\left(\zeta_{T}+3 \zeta_{n}\right) \tau}\right],
\end{aligned}
$$


where $a_{2}$ is related to the fourth moment of $\chi_{H}(\mathbf{c})$ through $\int d \mathbf{c} c^{4} \chi_{H}(\mathbf{c})=\frac{d(d+2)}{4}\left(1+a_{2}\right)$, and has been evaluated in the first Sonine approximation in [10]. All the functions $a_{\alpha, \beta}^{s}$ and $A_{\alpha, \beta}$ are evaluated in the Appendixes A and $\mathrm{B}$.

At this point, it is important to note that the Eqs. (59)-(62) have been obtained under the assumption that the system is in the homogeneous decay state at all times, i.e., that the one-particle distribution function is $\chi_{H}(\mathbf{c})$ for the whole time evolution. For $p<1$, if we start with an arbitrary initial condition, numerical simulations show that, after a few collisions, the distribution function reaches the scaling regime given by Eq. (18). Then, the evolution of $\phi_{H}^{(h)}$ is given by (53) and one expects that the same correlation functions (59)-(62) will be obtained in the long-time limit, independently of the initial condition. This will be confirmed in the next section by numerical simulations.

Equations (59)-(62) lead to a certain number of theoretical predictions. In particular, they imply that the ratios $\left\langle\delta N^{2}(\tau)\right\rangle / N_{H}(\tau),\left\langle\delta E^{2}(\tau)\right\rangle /\left[N_{H}(\tau) v_{H}^{4}(\tau)\right],\left\langle\delta P_{i}^{2}(\tau)\right\rangle /$ $\left[N_{H}(\tau) v_{H}^{2}(\tau)\right]$, and $\langle\delta N(\tau) \delta E(\tau)\rangle /\left[N_{H}(\tau) v_{H}^{2}(\tau)\right]$ reach stationary values at large times. The approach to these stationary values is exponential in $\tau$, and is slower for the correlations of the total momentum, since the argument of the exponential is $p\left(\zeta_{n}-\zeta_{T}\right) \tau$, while the other quantities evolve on faster time scales.

\section{Numerical simulations}

We now compare our theoretical predictions with the results of molecular dynamics (MD) and direct simulation Monte Carlo (DSMC) calculations of a freely evolving system of $N$ hard disks of diameter $\sigma$ which annihilate with probability $p$ or collide elastically with probability $1-p$ every time two particles meet each other. In the MD case, the particles were localized in a square box of size $L$ with periodic boundary conditions. The event-driven algorithm [19] has been used and the initial density has been chosen low enough to be always in the dilute limit. The parameters for all the MD simulations were $N(0)=10^{5}, n_{H}(0) \sigma^{2}=0.05$, $T_{H}(0)=1$, and $0<p \leq 1$. In the case of the DSMC simulations we have used Bird's algorithm [20] with the same values of the parameters, except that the density that plays no role. The initial velocity distribution is a Maxwellian in both cases. We have measured the time evolution of the total number of particles and the total energy, averaging the data over various initial conditions (the total momentum fluctuates around zero). We first checked that the Eqs. (20) and (21), with the theoretical predictions derived in [10] for the cooling rates, correctly describe the decay of the average global quantities. In the same way, we have obtained the averaged values of $N^{2}(t), E^{2}(t), P_{i}^{2}(t)$, and $N(t) E(t)$ (the correlations between $P_{i}$ and $N$ or $E$ are zero).

Figures 1-3 show the time evolution of the various onetime correlation functions considered, for $p=0.5$ and 0.8 . The DSMC results have been averaged over 4000 trajectories while the MD simulations have been averaged over 150 trajectories (the DSMC method being computationally less expensive, it is then possible to average over a larger number of initial conditions than for the MD simulations). The
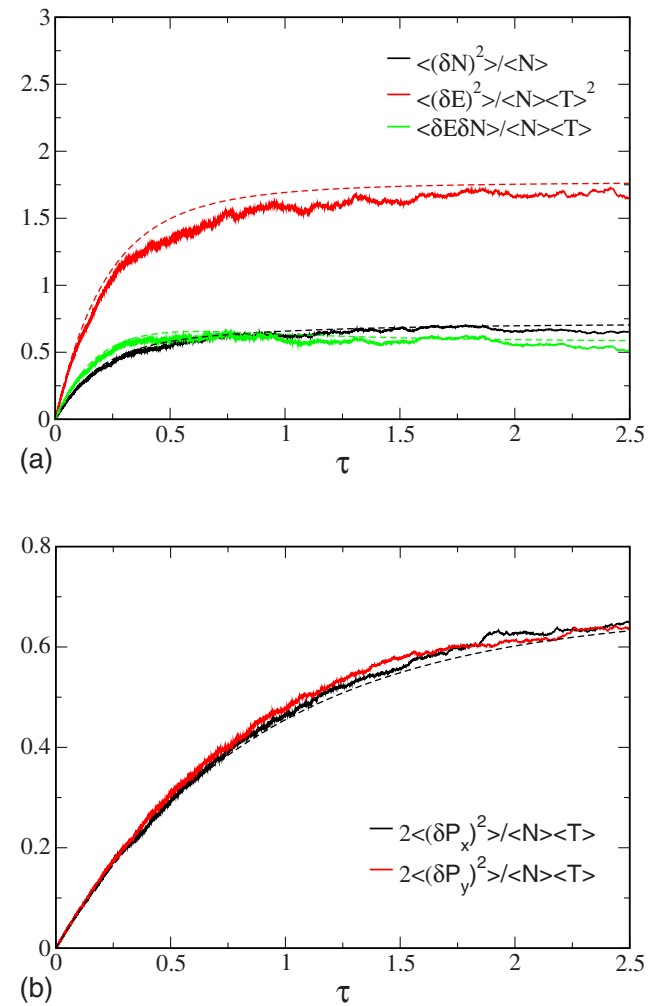

FIG. 1. (Color online) Scaled second moment of the fluctuations as a function of $\tau$ for a system with $p=0.8$. These results are from DSMC simulations and have been averaged over 4000 trajectories.

dashed lines are the theoretical predictions, Eqs. (59)-(62). Note, however, that the system is not initially in the HDS; the initial distribution function is a Maxwellian and not $\chi_{H}$. Nevertheless, as the difference between these two distributions is very small (at least for thermal velocities since $a_{2}$ $\sim 0.1$ ) and as the stationary values depend very weakly on $p$, Eqs. (59)-(62) predict quite well the time evolution measured in the simulations. The ratios $\left\langle\delta N^{2}(\tau)\right\rangle / N_{H}(\tau)$, $\left\langle\delta E^{2}(\tau)\right\rangle /\left[N_{H}(\tau) v_{H}^{4}(\tau)\right], \quad$ and $\quad\langle\delta N(\tau) \delta E(\tau)\rangle /\left[N_{H}(\tau) v_{H}^{2}(\tau)\right]$ reach stationary values as predicted. The fluctuations of the total momentum evolve more slowly, as also predicted, and the stationary value of $\left\langle\delta P_{i}^{2}(\tau)\right\rangle /\left[N_{H}(\tau) v_{H}^{2}(\tau)\right]$ is barely reached. Note that $\tau=4$ corresponds for $p=0.5$ to a total number of particles at the end of the simulation $N \simeq 1700$.

We have performed simulations starting with other initial conditions further from the HDS. The initial velocity distribution function has been chosen as a constant function in a square centered in the origin in the velocity space such that the initial temperature is unity. As seen in Figs. 4 and 5, we obtain a different short-time evolution but the scaled moments still converge toward the HDS values, which are represented by the dashed lines. The convergence is slower as we increase the value of $p$, and $\left\langle\delta E^{2}(\tau)\right\rangle /\left[N_{H}(\tau) v_{H}^{4}(\tau)\right]$, whose magnitude depends on the higher moments of the velocity distribution, is the most affected.

Figures 6 and 7 show the comparison between the stationary values of the various ratios measured in the simulations and the theoretical predictions in Eq. (59)-(62) at large $\tau$. The agreement is very good for all values investigated.

We have also computed the probability distribution for the number of particles, energy, and momentum. As we can see 

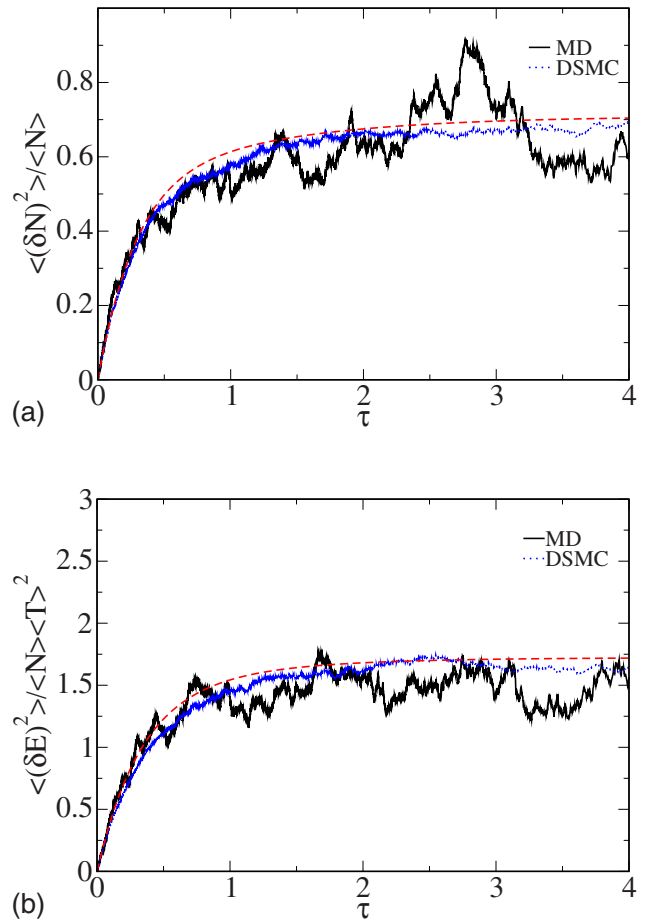

FIG. 2. (Color online) Second moment of the fluctuations of the number of particles (left panel) and of the total energy (right panel) as a function of the number of collisions per particle $\tau$, for a system with $p=0.5$ and initial number of particles $N=10^{5}$. The dashed lines are the theoretical predictions.

in Fig. 8, where we have considered a system with $p=0.5$, they are correctly described by a Gaussian distribution. The figure displays the distribution at four different times, showing that the shape of the probability distributions does not vary during the dynamical evolution. Similar results have been obtained for the probability distribution of the total momentum.

\section{TWO-TIME CORRELATION FUNCTION IN THE HDS}

In this section, we study the two-time correlation function of the global quantities in the HDS. With this aim, we consider two dynamical variables $A(t)$ and $B(t)$ as in (33), and compute the correlations $\left\langle\delta A(t) \delta B\left(t^{\prime}\right)\right\rangle_{H}$ for $t>t^{\prime}$, which are obtained from $h_{1,1, H}$ through Eq. (35).

As in the previous section, we start by integrating out the spatial degrees of freedom and consider

$$
\psi_{H}\left(\tau, \tau^{\prime}, \mathbf{c}_{1}, \mathbf{c}_{2}\right) \equiv \int d \mathbf{r}_{12} \widetilde{h}_{H}\left(\mathbf{r}_{12} ; \mathbf{c}_{1}, \tau ; \mathbf{c}_{2}, \tau^{\prime}\right),
$$

whose evolution equation is obtained by integrating (32) over space variables,

$$
\frac{\partial}{\partial \tau} \psi_{H}\left(\tau, \tau^{\prime}, \mathbf{c}_{1}, \mathbf{c}_{2}\right)=\Lambda\left(\mathbf{c}_{1}\right) \psi_{H}\left(\tau, \tau^{\prime}, \mathbf{c}_{1}, \mathbf{c}_{2}\right) .
$$

This equation has to be solved with the initial condition

$$
\psi_{H}\left(\tau^{\prime}, \tau^{\prime}, \mathbf{c}_{1}, \mathbf{c}_{2}\right)=\chi_{H}\left(\mathbf{c}_{1}\right) \delta\left(\mathbf{c}_{1}-\mathbf{c}_{2}\right)+\phi_{H}\left(\tau^{\prime}, \mathbf{c}_{1}, \mathbf{c}_{2}\right),
$$
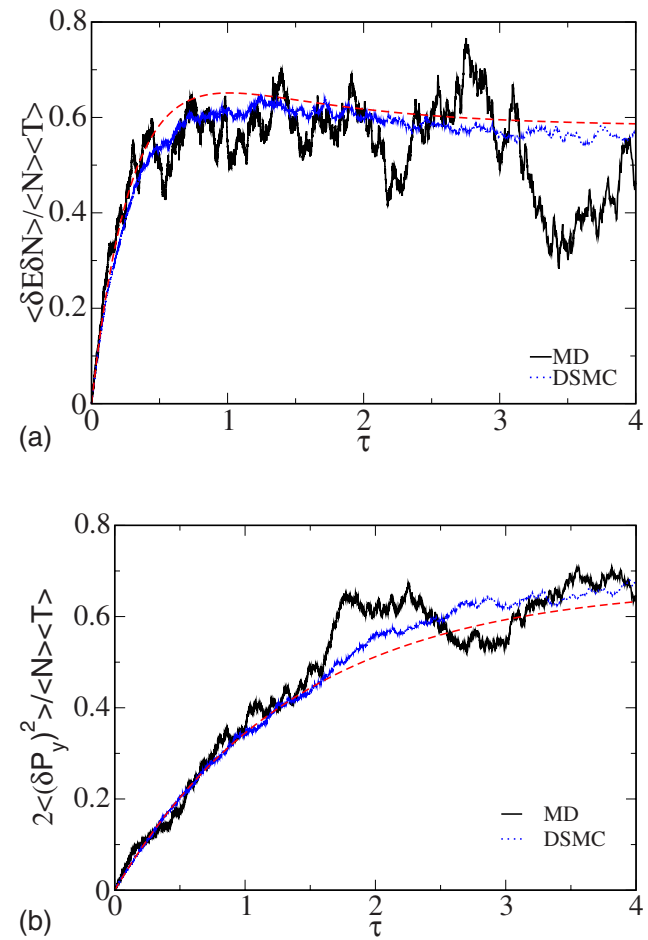

FIG. 3. (Color online) Correlation between the fluctuations of the total energy and total number of particles (left panel), and second moment of the fluctuations of the $y$ component of the total momentum (right panel), as a function of the number of collisions per particle $\tau$, for a system with $p=0.5$ and initial number of particles $N=10^{5}$. The dashed lines are the theoretical predictions.

where we have taken into account the scaling of $f_{H}(18)$ and $g_{2, H}(27)$. Then, using the approximation (52), we obtain

$$
\begin{aligned}
& P_{12} \psi_{H}\left(\tau, \tau^{\prime}, \mathbf{c}_{1}, \mathbf{c}_{2}\right) \\
&=e^{\Lambda\left(\mathbf{c}_{1}\right)\left(\tau-\tau^{\prime}\right)} P_{12} \psi_{H}\left(\tau^{\prime}, \tau^{\prime}, \mathbf{c}_{1}, \mathbf{c}_{2}\right) \\
&=\left\langle\bar{\xi}_{1}\left(\mathbf{c}_{1}\right) \mid \psi_{H}\left(\tau^{\prime}, \tau^{\prime}, \mathbf{c}_{1}, \mathbf{c}_{2}\right)\right\rangle \xi_{1}\left(\mathbf{c}_{1}\right) \\
& \\
&+\left\langle\bar{\xi}_{2}\left(\mathbf{c}_{1}\right) \mid \psi_{H}\left(\tau^{\prime}, \tau^{\prime}, \mathbf{c}_{1}, \mathbf{c}_{2}\right)\right\rangle \xi_{2}\left(\mathbf{c}_{1}\right) e^{-p\left(\zeta_{T}+2 \zeta_{n}\right)\left(\tau-\tau^{\prime}\right)} \\
&+\sum_{i}\left\langle\bar{\xi}_{3 i}\left(\mathbf{c}_{1}\right) \mid \psi_{H}\left(\tau^{\prime}, \tau^{\prime}, \mathbf{c}_{1}, \mathbf{c}_{2}\right)\right\rangle \xi_{3 i}\left(\mathbf{c}_{1}\right) e^{p \zeta_{T}\left(\tau-\tau^{\prime}\right)}
\end{aligned}
$$

Note that, assuming that the hydrodynamic eigenvalues are the biggest ones, we can deduce in the long-time limit that $P_{12} \psi_{H}\left(\tau, \tau^{\prime}\right)$ is a linear combination of the exponentials written above. Hence, the approximation (52) affects only the coefficients that multiply the exponentials, i.e., the initial conditions.

In the large-time limit $\tau, \tau^{\prime} \rightarrow \infty, \tau-\tau^{\prime}$ finite (and positive), we can replace $\psi_{H}\left(\tau^{\prime}, \tau^{\prime}, \mathbf{c}_{1}, \mathbf{c}_{2}\right)$ by $\chi_{H}\left(\mathbf{c}_{1}\right) \delta\left(\mathbf{c}_{1}-\mathbf{c}_{2}\right)$ $+\phi_{H}^{s}\left(\mathbf{c}_{1}, \mathbf{c}_{2}\right)$, so that

$$
\begin{aligned}
\left\langle\delta N(\tau) \delta N\left(\tau^{\prime}\right)\right\rangle_{H}= & N_{H}(\tau)\left(-A_{1,1}-z A_{1,2}-z A_{1,2}\right. \\
& \left.\times e^{-p\left(\zeta_{T}+2 \zeta_{n}\right)\left(\tau-\tau^{\prime}\right)}-z^{2} A_{2,2} e^{-p\left(\zeta_{T}+2 \zeta_{n}\right)\left(\tau-\tau^{\prime}\right)}\right),
\end{aligned}
$$



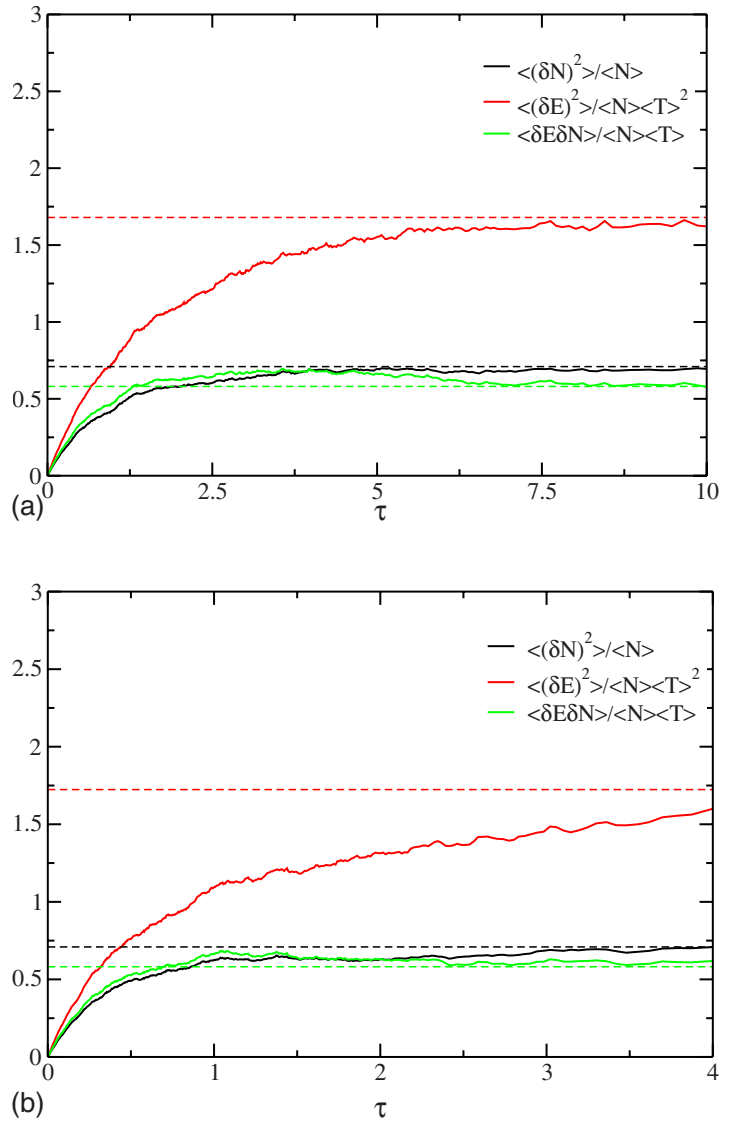

FIG. 4. (Color online) DSMC results for the scaled second moment of the fluctuations as a function of $\tau$ for systems with $p=0.2$ (left panel) and 0.5 (right panel). The dashed lines are the theoretical predictions for the stationary values. The initial velocity distribution at $\tau=0$ is uniform in a square domain.

$$
\begin{aligned}
\left\langle\delta P_{i}(\tau) \delta P_{j}\left(\tau^{\prime}\right)\right\rangle_{H}= & \delta_{i j} N_{H}(\tau) v_{H}(\tau) v_{H}\left(\tau^{\prime}\right)\left(a_{3 i, 3 i}^{s}+\frac{1}{2}\right) e^{p \zeta_{T}\left(\tau-\tau^{\prime}\right)}, \\
\left\langle\delta E(\tau) \delta E\left(\tau^{\prime}\right)\right\rangle_{H}= & \left(\frac{d m}{4}\right)^{2} N_{H}(\tau) v_{H}^{2}(\tau) v_{H}^{2}\left(\tau^{\prime}\right)\left\{-A_{1,1}\right. \\
& +(z+2) A_{1,2}+\left[-(z+2)^{2} A_{2,2}\right. \\
& \left.\left.+(z+2) A_{1,2}\right] e^{-p\left(\zeta_{T}+2 \zeta_{n}\right)\left(\tau-\tau^{\prime}\right)}\right\}, \\
\left\langle\delta N(\tau) \delta E\left(\tau^{\prime}\right)\right\rangle_{H}= & m \frac{d}{4} N_{H}(\tau) v_{H}^{2}\left(\tau^{\prime}\right)\left\{A_{1,1}-(z+2) A_{1,2}\right. \\
+ & {\left.\left[-z(z+2) A_{2,2}+z A_{1,2}\right] e^{-p\left(\zeta_{T^{+}}+2 \zeta_{n}\right)\left(\tau-\tau^{\prime}\right)}\right\} . }
\end{aligned}
$$

In the $\tau$ scale, it can be seen from Eqs. (20) and (21) that $N_{H}$ and $v_{H}$ decay exponentially. For $A, B=N, E, P$, the normalized correlation functions

$$
C_{A B}\left(\tau, \tau^{\prime}\right)=\left\langle\delta A(\tau) \delta B\left(\tau^{\prime}\right)\right\rangle /\left\langle\delta A\left(\tau^{\prime}\right) \delta B\left(\tau^{\prime}\right)\right\rangle
$$

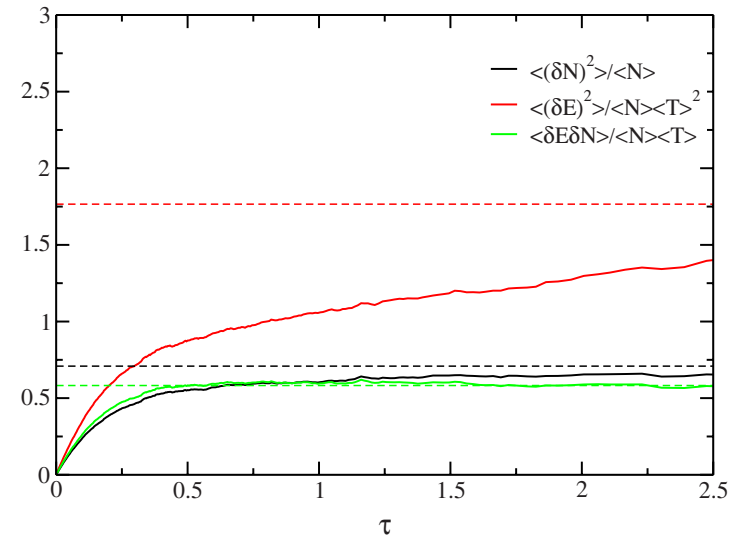

FIG. 5. (Color online) Same as in Fig. 4 but for a system with $p=0.8$.

become therefore time-translationally invariant, i.e., functions of $\tau-\tau^{\prime}$, once the stationary regime for the ratios such as $\left\langle\delta N^{2}(\tau)\right\rangle / N_{H}(\tau)$ has been reached (see the previous section). We have checked numerically that this is indeed the case, and we compare in Figs. 9 and 10 the evolution of $C_{N N}\left(\tau-\tau^{\prime}\right), C_{E E}\left(\tau-\tau^{\prime}\right)$, and $C_{N E}\left(\tau-\tau^{\prime}\right)$ measured in DSMC simulations (for $p=1$, averaged over 4000 trajectories) with the theoretical predictions. The agreement is very good. Figure 11 also shows the theoretical prediction for the decay of the momentum correlation function $C_{P P}\left(\tau, \tau^{\prime}\right)$ $=\left\langle\delta P_{i}(\tau) \delta P_{j}\left(\tau^{\prime}\right)\right\rangle /\left\langle\delta P_{i}\left(\tau^{\prime}\right) \delta P_{j}\left(\tau^{\prime}\right)\right\rangle$ for $p=0.5$. The characteristic decay time of $C_{P P}$ is of the order of $\tau \sim 4$. Because
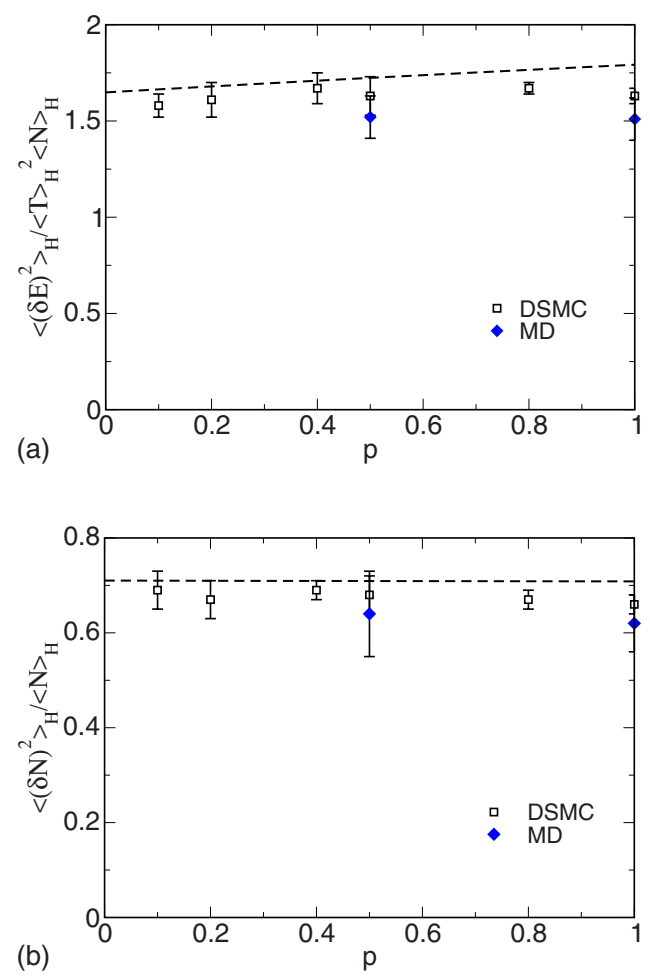

FIG. 6. (Color online) Average steady state of the scaled second moment of the fluctuations of the number of particles and of the energy fluctuations as a function of the annihilation probability $p$. The dashed lines show the large- $\tau$ predictions of Eqs. (59) and (61). 


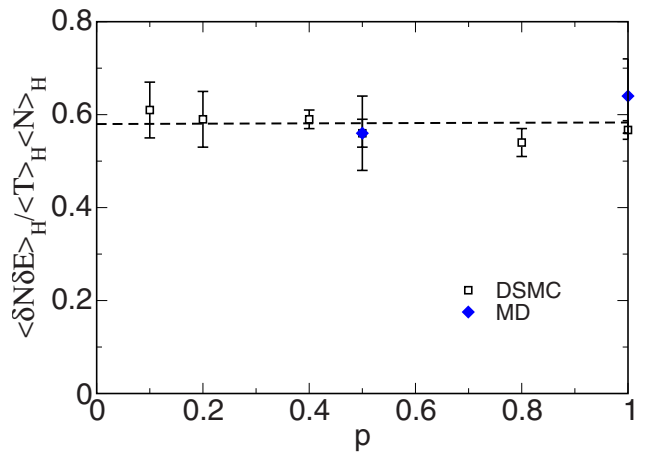

FIG. 7. (Color online) Average steady values of the one-time correlation of the energy and number of particle fluctuations as a function of the probability of annihilation $p$. The dashed line shows the large- $\tau$ prediction of Eq. (62).

the time to reach the stationary regime for $\left\langle\delta P_{i}^{2}(\tau)\right\rangle$ / $\left[N_{H}(\tau) v_{H}^{2}(\tau)\right]$ is $\tau \sim 4$, as shown in Fig. 3, we would need to reach $\tau \simeq 8$ in the numerical simulations in order to display numerical results for $C_{P P}$, which means that we would need to consider simulations with an initial number of particles of the order of $N(0) \sim 10^{7}$.

\section{CONCLUSIONS}

In this paper, we have formulated a general theory for fluctuations and correlations in a dilute probabilistic ballistic
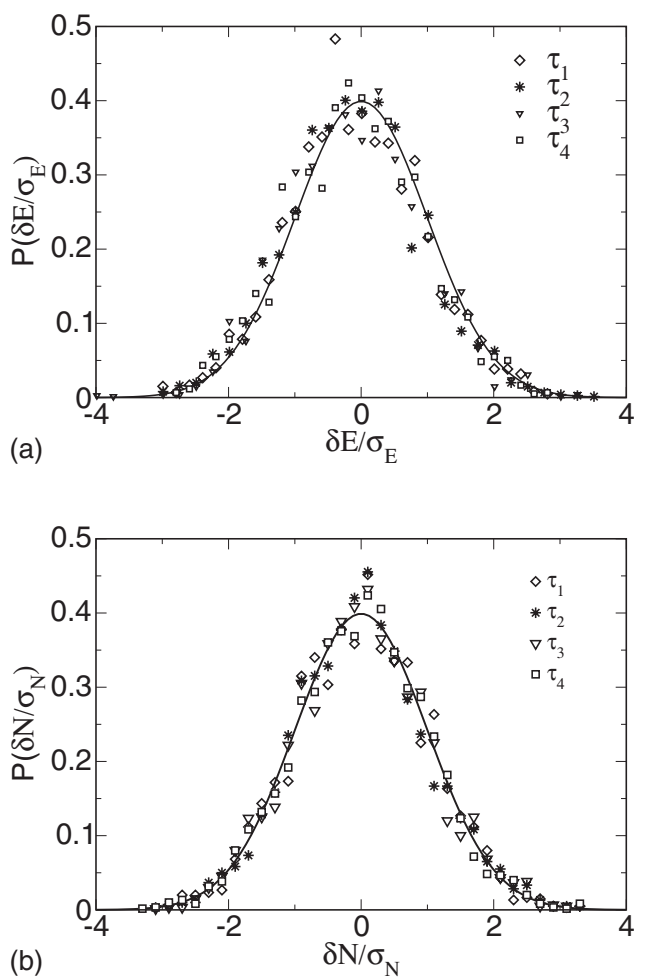

FIG. 8. Normalized distribution of the relative energy (left panel) and number of particle fluctuations (right panel) for a system with $p=0.5$. The symbols are from DSMC simulations and for four different values of $\tau, \tau_{1}=0.69, \tau_{2}=0.98, \tau_{3}=1.37$, and $\tau_{4}=2.05$. The solid line is a Gaussian with unit variance.
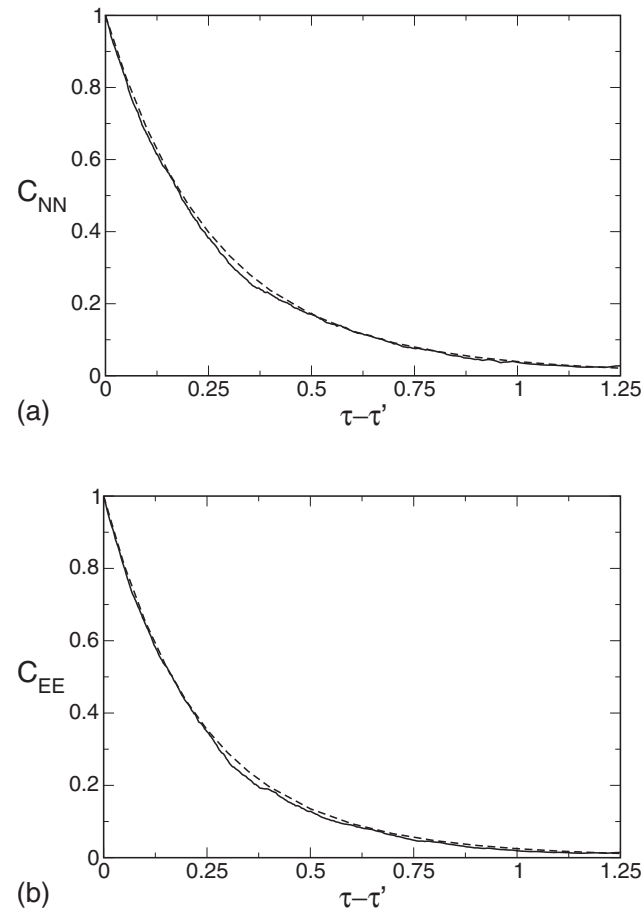

FIG. 9. Decay of the two-time correlation of the number of particles, $C_{N N}$, and the energy, $C_{E E}$, for a system with $p=1$, measured with DSMC simulations. The dashed line is the theoretical prediction.

annihilation system. The theory has been particularized to the homogeneous decay state, taking advantage of its scaling properties. For this state we have focused on the study of the fluctuations of the total number of particles, total momentum, and total energy, evaluating the two-time correlation functions between these quantities in the hydrodynamic approximation. The fluctuations of the total number of particles, total momentum, and total energy, once conveniently rescaled, converge to stationary values. The convergence is exponential in the natural time scale $\tau$ given by the number of collisions, and the corresponding rates are simple combinations of the cooling rates. The stationary values are obtained as functionals of the distribution function and can be computed in the first Sonine approximation. We have also

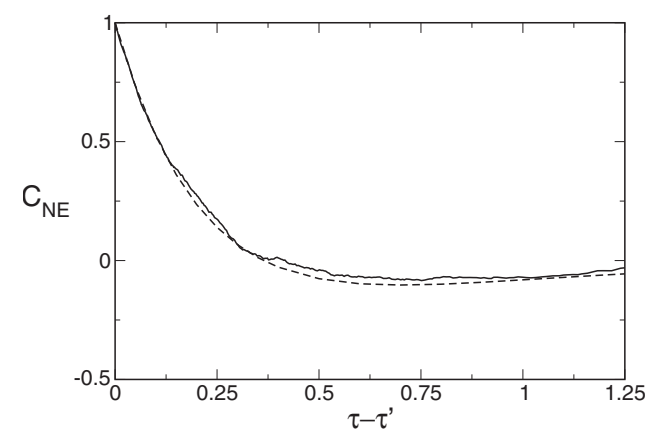

FIG. 10. Decay of the two-time correlation of the number of particles and the energy, $C_{N E}$, for a system with $p=1$. The dashed line is the theoretical prediction. 


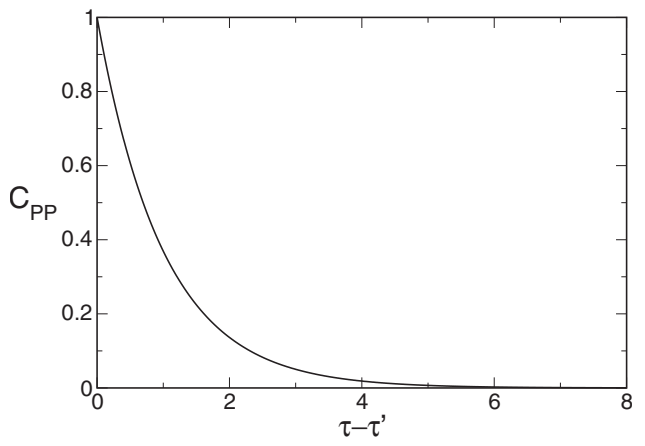

FIG. 11. Theoretical prediction for the decay of the momentum correlation function $C_{P P}\left(\tau, \tau^{\prime}\right)$, defined in the main text, as a function of $\tau-\tau^{\prime}$ for a system with $p=0.5$.

obtained theoretical expressions for the two-time correlations of global observables. All our theoretical predictions have been successfully compared with the results of molecular dynamics and DSMC numerical simulations, providing strong support for the theory developed here, including the hydrodynamic description in terms of the lowest-order eigenfunctions and eigenvalues of the linearized Boltzmann collision operator.

As a side remark, we note that the correlation functions contain two parts, one coming from the one-particle distribution function, and another one that comes from velocity correlations. Nevertheless, it must be stressed that the existence of these velocity correlations does not imply a violation of the "molecular chaos" assumption that underlies the Boltzmann equation. This is because the latter refers only to the precollisional part of the two-body distribution function (at contact).

The fact that the two-time correlation functions decay on a time scale determined by the cooling rates reflects the intuitive notion that their dynamic is essentially of macroscopic character, compatible with Onsager's regression hypothesis (see, e.g., [21]). To analyze this point in a deeper way, we show in Appendix $\mathrm{C}$ that a description of the fluctuations $\delta N, \delta E$, and $\delta P$ in terms of linear Langevin equations can be obtained, using for the deterministic part the evolution equations for a linear perturbation around the HDS. The noise terms in the Langevin equations (Gaussian and $\delta$-function correlated in time) can then be adjusted in order to obtain the same amplitudes for the one-time correlation functions as with our theory. In this respect, the results derived in Appendix $\mathrm{C}$ may be envisioned as formulating a fluctuation-dissipation theorem for the homogeneous decay state under scrutiny in this paper. The amplitudes of the noise terms are, however, complicated functions of moments of the one-particle distribution functions, and are not clearly related to macroscopic quantities such as the cooling rates.

\section{ACKNOWLEDGMENTS}

We would like to thank the Agence Nationale de la Recherche for financial support (Grant No. ANR-05-JCJC44482). MIGS acknowledges financial support from Becas de la Fundación La Caixa y el Gobierno Francés and from the HPC-EUROPA Project No. RII3-CT-2003-506079, with the support of the European Community Research Infrastructure Action. P.M. acknowledges financial support from the Programa José Castillejo of the Spanish Minister of Education and Science.

\section{APPENDIX A: EXPRESSIONS FOR $\boldsymbol{a}_{\beta_{1}, \beta_{2}}^{s}$}

In this appendix we compute the expressions for the coefficients $a_{\beta_{1}, \beta_{2}}^{s}$. Applying the projector $P_{12}$ to (40) yields, under the assumption $P_{12} \Lambda\left(\mathbf{c}_{i}\right)=P_{12} \Lambda\left(\mathbf{c}_{i}\right) P_{12}$,

$$
\begin{array}{r}
{\left[\Lambda\left(\mathbf{c}_{1}\right)+\Lambda\left(\mathbf{c}_{2}\right)-2 p \zeta_{n}\right] \phi_{H}^{s(h)}\left(\mathbf{c}_{1}, \mathbf{c}_{2}\right)} \\
=-\gamma P_{12} T\left(\mathbf{c}_{1}, \mathbf{c}_{2}\right) \chi_{H}\left(\mathbf{c}_{1}\right) \chi_{H}\left(\mathbf{c}_{2}\right) .
\end{array}
$$

Using the definition (51) of the coefficients $a_{\beta_{1}, \beta_{2}}^{s}$, we then obtain the set of equations

$$
\begin{gathered}
\sum_{\beta_{1}}^{3} \sum_{\beta_{2}}^{3} a_{\beta_{1}, \beta_{2}}^{s}\left(\lambda_{\beta_{1}}+\lambda_{\beta_{2}}-2 p \zeta_{n}\right) \xi_{\beta_{1}}\left(\mathbf{c}_{1}\right) \xi_{\beta_{2}}\left(\mathbf{c}_{2}\right) \\
=-P_{12} \gamma T\left(\mathbf{c}_{1}, \mathbf{c}_{2}\right) \chi_{H}\left(\mathbf{c}_{1}\right) \chi_{H}\left(\mathbf{c}_{2}\right),
\end{gathered}
$$

and it is straightforward to write

$$
a_{\beta_{1}, \beta_{2}}^{s}=-\frac{\left\langle\bar{\xi}_{\beta_{1}}\left(\mathbf{c}_{1}\right) \bar{\xi}_{\beta_{2}}\left(\mathbf{c}_{2}\right) \mid \gamma T\left(\mathbf{c}_{1}, \mathbf{c}_{2}\right) \chi_{H}\left(\mathbf{c}_{1}\right) \chi_{H}\left(\mathbf{c}_{2}\right)\right\rangle}{\lambda_{\beta_{1}}+\lambda_{\beta_{2}}-2 p \zeta_{n}} .
$$

Given the expression of the functions $\left\{\bar{\xi}_{i}(\mathbf{c})\right\}_{i=1}^{3}$, and taking into account the relations

$$
\begin{gathered}
p \zeta_{n}=-\frac{\gamma}{2} \int d \mathbf{c}_{1} \int d \mathbf{c}_{2} T\left(\mathbf{c}_{1}, \mathbf{c}_{2}\right) \chi_{H}\left(\mathbf{c}_{1}\right) \chi_{H}\left(\mathbf{c}_{2}\right), \\
p \zeta_{T}=-\frac{\gamma}{2} \int d \mathbf{c}_{1} \int d \mathbf{c}_{2}\left(\frac{2 c_{1}^{2}}{d}-1\right) T\left(\mathbf{c}_{1}, \mathbf{c}_{2}\right) \chi_{H}\left(\mathbf{c}_{1}\right) \chi_{H}\left(\mathbf{c}_{2}\right),
\end{gathered}
$$

we obtain

$$
\begin{aligned}
a_{1,1}^{s}= & -\left(1-\frac{z}{2(1+z)}\right)^{2}+\left(1+\frac{2}{z}\right) \frac{z}{1+z}\left(1-\frac{z}{2(1+z)}\right) \\
& +\frac{b(p)}{2 p \zeta_{n}} \frac{z^{2}}{(1+z)^{2}}, \\
a_{1,2}^{s}= & \frac{1}{p \zeta_{T}+4 p \zeta_{n}}\left[\left(\frac{z}{2(1+z)}-1\right) \frac{p\left(2 \zeta_{n}+\zeta_{T}\right)}{(1+z)}\right. \\
& \left.+p\left(\zeta_{n}+\zeta_{T}\right) \frac{z}{2(1+z)^{2}}-b(p) \frac{z}{(1+z)^{2}}\right], \\
a_{2,2}^{s}= & \frac{1}{2 p\left(\zeta_{T}+3 \zeta_{n}\right)}\left(-\frac{p\left(3 \zeta_{n}+2 \zeta_{T}\right)}{2(1+z)^{2}}+\frac{b(p)}{(1+z)^{2}}\right),
\end{aligned}
$$




$$
a_{3 i, 3 j}^{s}=\delta_{i j} \frac{c(p)}{2\left(\zeta_{T}-\zeta_{n}\right)},
$$

where

$$
b(p)=\gamma \int d \mathbf{c}_{1} \int d \mathbf{c}_{2} \frac{c_{1}^{2} c_{2}^{2}}{d^{2}} T\left(\mathbf{c}_{1}, \mathbf{c}_{2}\right) \chi_{H}\left(\mathbf{c}_{1}\right) \chi_{H}\left(\mathbf{c}_{2}\right),
$$

$$
\begin{aligned}
c(p)= & \gamma \int d \mathbf{c}_{1} \int d \mathbf{c}_{2} \chi_{H}\left(\mathbf{c}_{1}\right) \chi_{H}\left(\mathbf{c}_{2}\right) \int d \hat{\boldsymbol{\sigma}} \Theta\left(\mathbf{c}_{12} \cdot \hat{\boldsymbol{\sigma}}\right) \\
& \times\left(\mathbf{c}_{12} \cdot \hat{\boldsymbol{\sigma}}\right) c_{1 x} c_{2 x} .
\end{aligned}
$$

These two functions have been evaluated in first Sonine order, with the result

$$
b(p)=-\frac{16[-1+4 d(d+1)] p+a_{2}\{256-255 p+4 d[-64+(71+7 d) p]\}}{128 \sqrt{2} d^{2} \Gamma(d / 2)} \pi^{(d-1) / 2} \gamma
$$

$$
c(p)=\frac{(-16+5 a 2)}{32 \sqrt{2} d \Gamma(d / 2)} \pi^{(d-1) / 2} \gamma .
$$

\section{APPENDIX B: EXPRESSIONS FOR $\boldsymbol{a}_{\boldsymbol{\beta}_{1}, \boldsymbol{\beta}_{2}}(\mathbf{0})$}

In this appendix we evaluate the coefficient $a_{\beta_{1}, \beta_{2}}(0)$ for the specific case in which we have

$$
\begin{aligned}
\left\langle\delta N^{2}(0)\right\rangle & =0, \quad\left\langle\delta P_{i}(0) \delta P_{j}(0)\right\rangle=0, \\
\left\langle\delta E^{2}(0)\right\rangle & =0, \quad\langle\delta N(0) \delta E(0)\rangle=0 .
\end{aligned}
$$

Taking these relations into account, it is straightforward to obtain

$$
\begin{gathered}
\int d \mathbf{c}_{1} \int d \mathbf{c}_{2} \phi_{H}\left(0, \mathbf{c}_{1}, \mathbf{c}_{2}\right)=-1 \\
\int d \mathbf{c}_{1} \int d \mathbf{c}_{2} c_{i i} c_{2 j} \phi_{H}\left(0, \mathbf{c}_{1}, \mathbf{c}_{2}\right)=-\frac{1}{2} \delta_{i j}, \\
\int d \mathbf{c}_{1} \int d \mathbf{c}_{2} c_{2}^{2} \phi_{H}\left(0, \mathbf{c}_{1}, \mathbf{c}_{2}\right)=-\frac{d}{2}, \\
\int d \mathbf{c}_{1} \int d \mathbf{c}_{2} c_{1}^{2} c_{2}^{2} \phi_{H}\left(0, \mathbf{c}_{1}, \mathbf{c}_{2}\right)=-\frac{d(d+2)}{4}\left(1+a_{2}\right) .
\end{gathered}
$$

With these expressions and the definition of $\bar{\xi}_{i}$, we get

$$
\begin{gathered}
a_{1,1}(0)=\frac{1}{(1+z)^{2}}\left(\frac{z}{2}(z+2)-\frac{1}{4}(z+2)^{2}-\frac{d+2}{4 d} z^{2}\left(1+a_{2}\right)\right), \\
a_{1,2}(0)=\frac{1}{2(1+z)^{2}}\left[-\left(\frac{z}{2}+2\right)+\frac{d+2}{2 d} z\left(1+a_{2}\right)\right],
\end{gathered}
$$

$$
\begin{gathered}
a_{2,2}(0)=-\frac{1}{(1+z)^{2}}\left(\frac{3}{4}+\frac{d+2}{4 d}\left(1+a_{2}\right)\right), \\
a_{3 i, 3 i}(0)=-\frac{1}{2} .
\end{gathered}
$$

\section{APPENDIX C: LANGEVIN EQUATIONS FOR THE GLOBAL MAGNITUDES}

In this appendix, we will show that it is possible to find a Langevin description for the fluctuations of the global magnitudes of the system. The idea is to assume that the global magnitudes obey some equations that can be decomposed into a deterministic part, which is identified with the macroscopic equations for a linear perturbation of the HDS, plus a Gaussian white noise. Because of formulas (59)-(62) let us study the equations for the magnitudes

$$
\begin{gathered}
\delta \tilde{N}(\tau)=\frac{\delta N(\tau)}{N_{H}^{1 / 2}(\tau)}, \quad \delta \tilde{P}_{i}(\tau)=\frac{\delta P_{i}(\tau)}{N_{H}^{1 / 2}(\tau) v_{H}(\tau)}, \\
\delta \tilde{E}(\tau)=\frac{4 \delta E(\tau)}{d m N_{H}^{1 / 2}(\tau) v_{H}^{2}(\tau)},
\end{gathered}
$$

in order to deal with processes with time-independent variances.

Let us start with the easiest one, the equation for $\delta \widetilde{P}_{i}(\tau)$. We can define the function

$$
\omega_{i, \mathbf{k}=\mathbf{0}}(\tau)=\frac{1}{n_{H}(t) v_{H}(t)} \int d \mathbf{r} \int d \mathbf{v} v_{i} \delta f(\mathbf{r}, \mathbf{v}, t)
$$

where $\delta f(\mathbf{r}, \mathbf{v}, t) \equiv f(\mathbf{r}, \mathbf{v}, t)-f_{H}(\mathbf{v}, t)$, with $f_{H}(\mathbf{v}, t)$ the distribution function in the HDS. Then, if the generic distribution function $f(\mathbf{r}, \mathbf{v}, t)$ is close enough to the HDS one, the linear equation for $\omega_{i, \mathbf{k}=\mathbf{0}}(\tau)$ is (see the preceding paper) 


$$
\left(\frac{\partial}{\partial \tau}-p \zeta_{T}\right) \omega_{i, \mathbf{k}=\mathbf{0}}(\tau)=0
$$

Then it is straightforward to see that the equation for the macroscopic deviation $\delta \widetilde{P}^{M}$ would be

$$
\left(\frac{\partial}{\partial \tau}+p\left(\zeta_{n}-\zeta_{T}\right)\right) \delta \widetilde{P}^{M}(\tau)=0
$$

where the superscript $M$ denotes macroscopic. Now, let us suppose that the equation for the fluctuating $\delta \widetilde{P}$ is of the form

$$
\left(\frac{\partial}{\partial \tau}+p\left(\zeta_{n}-\zeta_{T}\right)\right) \delta \tilde{P}_{i}(\tau)=R_{p}(\tau)
$$

with $R_{p}(\tau)$ a Gaussian white noise with the following properties:

$$
\left\langle R_{p}(\tau)\right\rangle_{H}=0, \quad\left\langle R_{p}(\tau) R_{p}\left(\tau^{\prime}\right)\right\rangle_{H}=\Gamma_{p} \delta\left(\tau-\tau^{\prime}\right) .
$$

That is, we consider that the equation describing the dynamics of the fluctuations can be obtained from the macroscopic equation describing the evolution of the system. Under these hypotheses we can calculate $\left\langle\delta \widetilde{P}(\tau) \delta \widetilde{P}\left(\tau^{\prime}\right)\right\rangle$. The solution of Eq. (C5) in the long-time limit is

$$
\delta \widetilde{P}_{i}(\tau)=\int_{0}^{\tau} d \tau^{\prime} e^{-p\left(\zeta_{n}-\zeta_{T}\right)\left(\tau-\tau^{\prime}\right)} R_{p}\left(\tau^{\prime}\right)
$$

and the autocorrelation function is, for $\tau>\tau^{\prime}$,

$$
\left\langle\delta \tilde{P}(\tau) \delta \tilde{P}\left(\tau^{\prime}\right)\right\rangle=\frac{\Gamma_{p} e^{-p\left(\zeta_{n}-\zeta_{T}\right)\left(\tau-\tau^{\prime}\right)}}{2 p\left(\zeta_{n}-\zeta_{T}\right)}\left(1-e^{-2 p\left(\zeta_{n}-\zeta_{T}\right) \tau^{\prime}}\right)
$$

We are interested in the limit $\tau^{\prime} \rightarrow \infty, \tau \rightarrow \infty, \tau^{\prime}-\tau \sim$ finite. In this limit we obtain

$$
\left\langle\delta \tilde{P}(\tau) \delta \tilde{P}\left(\tau^{\prime}\right)\right\rangle_{H}=\frac{\Gamma_{p}}{2 p\left(\zeta_{n}-\zeta_{T}\right)} e^{-p\left(\zeta_{n}-\zeta_{T}\right)\left(\tau-\tau^{\prime}\right)} .
$$

Now one can relate this result to the one obtained previously, Eq. (68), which can be expressed in our variables as

$$
\left\langle\delta \tilde{P}(\tau) \delta \tilde{P}\left(\tau^{\prime}\right)\right\rangle_{H}=\left(\frac{1}{2}+a_{3 i, 3 i}^{s}\right) e^{-p\left(\zeta_{n}-\zeta_{T}\right)\left(\tau-\tau^{\prime}\right)} .
$$

Comparing Eqs. (C9) and (C10), it is seen that if

$$
\Gamma_{p}=2 p\left(\zeta_{n}-\zeta_{T}\right)\left(\frac{1}{2}+a_{3 i, 3 i}^{s}\right)
$$

the Langevin equation (C5) is in agreement with the results obtained in the previous section.

Now we will sketch the derivation of the Langevin equations for the other fluctuating quantities. First of all, we are going to start from the macroscopic equation for $\rho_{0}$ and $\varepsilon_{0}$ defined as

$$
\begin{gathered}
\rho_{\mathbf{0}} \equiv \rho_{\mathbf{k}=\mathbf{0}}(\tau)=\frac{1}{n_{H}(t)} \int d \mathbf{r} \int d \mathbf{v} \delta f(\mathbf{r}, \mathbf{v}, t), \\
\varepsilon_{\mathbf{0}} \equiv \varepsilon_{\mathbf{k}=\mathbf{0}}(\tau)=\frac{2}{d n_{H}(t) T_{H}(t)} \int d \mathbf{r} \int d \mathbf{v} \frac{1}{2} m v^{2} \delta f(\mathbf{r}, \mathbf{v}, t) .
\end{gathered}
$$

These equations are

$$
\frac{\partial}{\partial \tau} \rho_{0}(\tau)+p \zeta_{n}\left[\rho_{0}(\tau)+\varepsilon_{0}(\tau)\right]=0,
$$

$$
\frac{\partial}{\partial \tau} \varepsilon_{0}(\tau)+p\left(\zeta_{n}+\zeta_{T}\right)\left[\rho_{0}(\tau)+\varepsilon_{0}(\tau)\right]=0,
$$

from which we can write the equations for $\delta \tilde{N}^{M}$ $=V^{-1 / 2} n_{H}^{1 / 2} \rho_{0}$ and $\delta \widetilde{E}^{M}=V^{-1 / 2} n_{H}^{1 / 2} \varepsilon_{0}$ :

$$
\begin{gathered}
\frac{\partial}{\partial \tau} \delta \widetilde{N}^{M}=-2 p \zeta_{n} \delta \widetilde{N}^{M}-p \zeta_{n} \delta \widetilde{E}^{M} \\
\frac{\partial}{\partial \tau} \delta \widetilde{E}^{M}=-p\left(\zeta_{n}+\zeta_{T}\right) \delta \widetilde{N}^{M}-p\left(2 \zeta_{n}+\zeta_{T}\right) \delta \widetilde{E}^{M} .
\end{gathered}
$$

As we obtain a linear system of coupled equations, it is convenient to introduce some new variables to diagonalize the problem:

$$
\begin{gathered}
X_{1}^{M}=\frac{\zeta_{n}+\zeta_{T}}{\zeta_{n}} \delta \widetilde{N}^{M}-\delta \widetilde{E}^{M} \\
X_{2}^{M}=\delta \tilde{N}^{M}+\delta \widetilde{E}^{M}
\end{gathered}
$$

We obtain

$$
\begin{gathered}
\frac{\partial}{\partial \tau} X_{1}^{M}=-p \zeta_{n} X_{1}^{M}, \\
\frac{\partial}{\partial \tau} X_{2}^{M}=-p\left(3 \zeta_{n}+\zeta_{T}\right) X_{2}^{M} .
\end{gathered}
$$

Let us suppose now that the equations for $X_{i}(\tau)$ have the form of the macroscopic equations (C20) and (C21) plus a Gaussian random noise whose variance has to be computed to reproduce the results that we have obtained for the correlation function of the fluctuations of $N$ and $E$. The equations for the fluctuating variables $X_{1}$ and $X_{2}$ are thus

$$
\left(\frac{\partial}{\partial \tau}+p \zeta_{n}\right) X_{1}=R_{1}(\tau)
$$

$$
\left(\frac{\partial}{\partial \tau}+p\left(3 \zeta_{n}+\zeta_{T}\right)\right) X_{2}=R_{2}(\tau)
$$

Then, if we suppose that the noise terms have zero mean and their correlation function is $\delta$-function correlated in time, 


$$
\left\langle R_{i}(\tau)\right\rangle_{H}=0, \quad\left\langle R_{i}\left(\tau^{\prime}\right) R_{j}(\tau)\right\rangle_{H}=\Gamma_{i j} \delta\left(\tau^{\prime}-\tau\right),
$$

we can obtain the values of the amplitudes of the noise term $\Gamma_{i j}$ in the same way we have done with the momentum, that is, by comparing with the results we obtained in the previous section. We obtain

$$
\begin{gathered}
\Gamma_{11}=-8 p \zeta_{n} \frac{(1+z)^{2}}{z^{2}} A_{11}, \\
\Gamma_{22}=-8 p\left(3 \zeta_{n}+\zeta_{T}\right)(1+z)^{2} A_{22},
\end{gathered}
$$

$$
\Gamma_{12}=-4 p\left(4 \zeta_{n}+\zeta_{T}\right) \frac{(1+z)^{2}}{z} A_{12}
$$

These calculations show that it is possible to describe the dynamics of fluctuations in the HDS in terms of some Langevin equations with Gaussian white noise. Due to the Gaussian nature of the noise and given that the equations are linear, the probability distribution function for those processes will also be Gaussian, in agreement with our simulations. It is worth pointing out that, although the amplitudes of the noises are known, they are not related in a simple way to the cooling rates $\zeta_{n}$ and $\zeta_{T}$ that appear in the deterministic part of the equations.
[1] M. I. García de Soria et al., preceding paper, Phys. Rev. E 77, 051127 (2008).

[2] M. Bramson and J. L. Lebowitz, Phys. Rev. Lett. 61, 2397 (1988).

[3] A. Ovchinnikov and Y. Zeldovitch, Chem. Phys. 28, 215 (1978).

[4] D. Toussaint and F. Wilczek, J. Chem. Phys. 78, 2642 (1983).

[5] S. Redner and K. Kang, Phys. Rev. Lett. 51, 1729 (1983).

[6] F. Leyvraz, Phys. Rep. 383, 95 (2003).

[7] F. Coppex, M. Droz, and E. Trizac, Phys. Rev. E 69, 011303 (2004).

[8] P. L. Krapivsky and C. Sire, Phys. Rev. Lett. 86, 2494 (2001).

[9] E. Trizac, Phys. Rev. Lett. 88, 160601 (2002).

[10] J. Piasecki, E. Trizac, and M. Droz, Phys. Rev. E 66, 066111 (2002).

[11] A. Lipowski, D. Lipowska, and A. L. Ferreira, Phys. Rev. E 73, 032102 (2006).

[12] F. Coppex, M. Droz, and E. Trizac, Phys. Rev. E 70, 061102 (2004).
[13] T. P. C. van Noije, M. H. Ernst, R. Brito, and J. A. G. Orza, Phys. Rev. Lett. 79, 411 (1997).

[14] J. J. Brey, F. Moreno, and M. J. Ruiz-Montero, Phys. Fluids 10, 2965 (1998).

[15] J. J. Brey, M. I. García de Soria, P. Maynar, and M. J. RuizMontero, Phys. Rev. Lett. 94, 098001 (2005).

[16] J. J. Brey, A. Domínguez, M. I. García de Soria, and P. Maynar, Phys. Rev. Lett. 96, 158002 (2006).

[17] M. H. Ernst and E. G. D. Cohen, J. Stat. Phys. 25, 153 (1981)

[18] J. J. Brey, M. I. García de Soria, P. Maynar, and M. J. RuizMontero, Phys. Rev. E 70, 011302 (2004).

[19] M. P. Allen and D. J. Tildesley, Computer Simulation of Liquids (Oxford Science Publications, Bristol, 1987).

[20] G. A. Bird, Molecular Gas Dynamics and the Direct Simulation of Gas Flows (Clarendon, Oxford, 1994).

[21] D. Chandler, Introduction to Modern Statistical Mechanics (Oxford University Press, New York, 1987). 